\title{
ASYMPTOTIC STRUCTURE OF FREE PRODUCT VON NEUMANN ALGEBRAS
}

\author{
CYRIL HOUDAYER AND YOSHIMICHI UEDA
}

\begin{abstract}
Let $(M, \varphi)=\left(M_{1}, \varphi_{1}\right) *\left(M_{2}, \varphi_{2}\right)$ be the free product of any $\sigma$-finite von Neumann algebras endowed with any faithful normal states. We show that whenever $Q \subset M$ is a von Neumann subalgebra with separable predual such that both $Q$ and $Q \cap M_{1}$ are the ranges of faithful normal conditional expectations and such that both the intersection $Q \cap M_{1}$ and the central sequence algebra $Q^{\prime} \cap M^{\omega}$ are diffuse (e.g. $Q$ is amenable), then $Q$ must sit inside $M_{1}$. This result generalizes the previous results of the first named author in Ho14 and moreover completely settles the questions of maximal amenability and maximal property Gamma of the inclusion $M_{1} \subset M$ in arbitrary free product von Neumann algebras.
\end{abstract}

\section{Introduction AND StATEMEnt of THE MAIN RESUlts}

The first class of concrete maximal amenable subalgebras in von Neumann algebras was discovered by Popa in his seminal article Po83. He showed that the generator maximal abelian subalgebra $\mathrm{L}(\mathbf{Z})=\mathrm{L}(\langle a\rangle)$ is maximal amenable inside the free group factor $\mathrm{L}\left(\mathbf{F}_{2}\right)=\mathrm{L}(\langle a, b\rangle)$. Popa moreover introduced in Po83] a powerful method, based on the study of central sequences in the ultraproduct framework, to prove that a given amenable von Neumann subalgebra in a finite von Neumann algebra is maximal amenable. This method will be referred to as Popa's asymptotic orthogonality property in this paper. Popa's maximal amenability result [Po83] for free group factors was recently extended by the first named author in [Ho14 to a large class of free product von Neumann algebras, possibly of type III. We refer to [Ho14] and the references therein for further results on maximal amenability in the framework of von Neumann algebras. We point out that Boutonnet-Carderi recently introduced in [BC14] a new method, based on the study of central states, to prove that a given amenable von Neumann subalgebra in a finite von Neumann algebra is maximal amenable. Among other things, they obtained concrete examples of maximal amenable von Neumann subalgebras in type $\mathrm{II}_{1}$ factors associated with higher rank lattices.

The aim of this paper is to further generalize the recent work of the first named author in [Ho14] and to completely settle the questions of maximal amenability and maximal property Gamma of the inclusion $M_{1} \subset M$ arising from an arbitrary free product $(M, \varphi)=\left(M_{1}, \varphi_{1}\right) *\left(M_{2}, \varphi_{2}\right)$.

We will say that an inclusion of von Neumann algebras $Q \subset M$ is with expectation if there exists a faithful normal conditional expectation from $M$ onto $Q$. Our first main result is the following optimal Gamma stability result inside arbitrary free product von Neumann algebras.

Theorem A. For each $i \in\{1,2\}$, let $\left(M_{i}, \varphi_{i}\right)$ be any $\sigma$-finite von Neumann algebra endowed with a faithful normal state. Denote by $(M, \varphi)=\left(M_{1}, \varphi_{1}\right) *\left(M_{2}, \varphi_{2}\right)$ the free product. Let $Q \subset M$ be any von Neumann subalgebra with separable predual and with expectation such that $Q \cap M_{1}$ is diffuse and with expectation, and furthermore that $Q^{\prime} \cap M^{\omega}$ is diffuse. Then we have $Q \subset M_{1}$.

2010 Mathematics Subject Classification. 46L10, 46L54, 46L36.

Key words and phrases. Asymptotic orthogonality property; Free product von Neumann algebras; Popa's deformation/rigidity theory; Type III factors; Ultraproduct von Neumann algebras.

CH is supported by ANR grant NEUMANN and ERC Starting Grant GAN 637601.

YU is supported by Grant-in-Aid for Scientific Research (C) 24540214. 
We refer to Theorem 4.3 below for a more general statement that extend [Ho14, Theorem D] to arbitrary free product von Neumann algebras. As a corollary to Theorem $\mathrm{A}$, we infer that amenable von Neumann subalgebras $Q \subset M$ with expectation such that the intersection $Q \cap M_{1}$ is diffuse and with expectation must in fact sit inside $M_{1}$. Namely, we obtain the following result.

Corollary B. Let $(M, \varphi)=\left(M_{1}, \varphi_{1}\right) *\left(M_{2}, \varphi_{2}\right)$ be as in Theorem A. Let $Q \subset M$ be any amenable von Neumann subalgebra with expectation such that $Q \cap M_{1}$ is diffuse and with expectation. Then we have $Q \subset M_{1}$.

We point out that the separability assumption on the predual of the amenable von Neumann subalgebra $Q \subset M$ is no longer needed in Corollary B. As we mentioned before, in the case when both $M_{1}$ and $M_{2}$ are tracial and both $\varphi_{1}$ and $\varphi_{2}$ are faithful normal tracial states, Corollary B is a consequence of [Ho14, Theorem D]. Very recently, Ozawa gave in Oz15] a short proof of the above Corollary B in the tracial case using an idea in [BC14]. However, that proof depends upon the assumption that given states are tracial. Moreover, we would like to emphasize that the tools and the techniques we will develop in order to achieve the goal of this paper have strong potential in future research, and indeed lead to our next work on general rigidity phenomenon for free product von Neumann algebras [HU15.

We also point out that [Ho14, Theorem A and Corollary B] hold true under the more general assumption that $M_{1}$ is diffuse, instead of the centralizer $\left(M_{1}\right)^{\varphi_{1}}$ being diffuse as in [Ho14]. In fact, we prove the optimal asymptotic orthogonality property result in arbitrary free product von Neumann algebras (see Theorem 3.1 below) to make those assertions hold under such a general assumption. Remark that this generalization of [Ho14, Theorem A] does not follow from Theorem $\mathrm{A}$, since it is applicable to any intermediate subalgebra $M_{1} \subset Q \subset M$ without a priori assuming it to be with expectation.

We now briefly explain the strategy of the proof of Theorem $\mathrm{A}$. To simplify the discussion, we will further assume that $Q \subset M$ is a subfactor. We refer to Section 4 for further details.

Assume that $Q$ is amenable. In that case, we exploit the fact that $Q$ is $\operatorname{AFD}$ with a Cartan subalgebra $A \subset Q$ and hence has lots of central sequences that sit inside the ultraproduct von Neumann subalgebra $A^{\omega} \subset Q^{\omega}$. This is a key observation when $Q$ is of type III. Using our generalization of the asymptotic orthogonality property in arbitrary free product von Neumann algebras (see Theorem 3.1 below) and exploiting the recent generalization of Popa's intertwining techniques obtained in [HI15], we then show that any corner of $A$ must embed with expectation into $M_{1}$ inside $M$. By exploiting the regularity property of the Cartan inclusion $A \subset Q$ and using a standard maximality argument, we deduce that $Q \subset M_{1}$.

We point out that our strategy, based on the study of central sequences in the ultraproduct framework via Popa's asymptotic orthogonality property, works for arbitrary von Neumann algebras. Hence we are able to deal with amenable subfactors $Q \subset M$ in Theorem $\mathrm{A}$ and Corollary B that can possibly be of type III.

Assume that $Q$ is nonamenable. In that case, we use Connes-Takesaki's structure theory [Co72, Ta03] and Popa's deformation/rigidity theory [Po01, Po03, Po06] inside the ultraproduct of the continuous core $\left(\mathrm{c}_{\varphi}(M)\right)^{\omega}$. One of the new features of our proof is to exploit a recent result of Masuda-Tomatsu [MT13] showing that the continuous core of the ultraproduct von Neumann algebra $c_{\varphi^{\omega}}\left(M^{\omega}\right)$ sits, as an intermediate von Neumann subalgebra with trace preserving conditional expectations, between $\mathrm{c}_{\varphi}(M)$ and $\left(\mathrm{c}_{\varphi}(M)\right)^{\omega}$, that is,

$$
\mathrm{c}_{\varphi}(M) \subset \mathrm{c}_{\varphi^{\omega}}\left(M^{\omega}\right) \subset\left(\mathrm{c}_{\varphi}(M)\right)^{\omega} .
$$

Using Popa's spectral gap rigidity principle and intertwining techniques, we then show that any finite corner of $\mathrm{c}_{\varphi}(Q)$ must embed into $\mathrm{c}_{\varphi}\left(M_{1}\right)$ inside the ambient continuous core $\mathrm{c}_{\varphi}(M)$. By a standard maximality argument, we deduce that $\mathrm{c}_{\varphi}(Q) \subset \mathrm{c}_{\varphi}\left(M_{1}\right)$ and hence $Q \subset M_{1}$. 
We point out that we do need to pass to the continuous core $c_{\varphi}(M)$ in order to make Popa's spectral gap rigidity principle work since we ultimately use Connes's characterization of amenability for finite von Neumann algebras [Co75.

We conclude this paper with an appendix in which we give a short proof of an unpublished result due to the second named author showing that Connes's bicentralizer problem has a positive solution for all type $\mathrm{III}_{1}$ factors arising as free products of von Neumann algebras.

\section{COnTEnTs}

1. Introduction and statement of the main results

2. Preliminaries

3. Asymptotic orthogonality property

4. Proofs of Theorem $\mathrm{A}$ and Corollary B

Appendix A. Bicentralizer problem for free product von Neumann algebras

References

\section{Preliminaries}

For any von Neumann algebra $M$, we will denote by $\mathcal{Z}(M)$ the centre of $M$, by $\mathcal{U}(M)$ the group of unitaries in $M$, by $\operatorname{Ball}(M)$ the unit ball of $M$ with respect to the uniform norm $\|\cdot\|_{\infty}$ and by $\left(M, \mathrm{~L}^{2}(M), J^{M}, \mathfrak{P}^{M}\right)$ the standard form of $M$. More generally, for any linear subspace $\mathcal{V} \subset M$, we will denote by $\operatorname{Ball}(\mathcal{V})$ the unit ball of $\mathcal{V}$ with respect to the uniform norm $\|\cdot\|_{\infty}$.

Background on $\sigma$-finite von Neumann algebras. Let $M$ be any $\sigma$-finite von Neumann algebra with unique predual $M_{*}$ and $\varphi \in M_{*}$ any faithful state. We will write $\|x\|_{\varphi}=\varphi\left(x^{*} x\right)^{1 / 2}$ for all $x \in M$. Recall that on $\operatorname{Ball}(M)$, the topology given by $\|\cdot\|_{\varphi}$ coincides with the $\sigma$-strong topology. Denote by $\xi_{\varphi} \in \mathfrak{P}^{M}$ the unique canonical implementing vector of $\varphi$. The mapping $M \rightarrow \mathrm{L}^{2}(M): x \mapsto x \xi_{\varphi}$ defines an embedding with dense image such that $\|x\|_{\varphi}=\left\|x \xi_{\varphi}\right\|_{\mathrm{L}^{2}(M)}$ for all $x \in M$.

We denote by $\sigma^{\varphi}$ the modular automorphism group of the state $\varphi$. The centralizer $M^{\varphi}$ of the state $\varphi$ is by definition the fixed point algebra of $\left(M, \sigma^{\varphi}\right)$. The continuous core of $M$ with respect to $\varphi$, denoted by $\mathrm{c}_{\varphi}(M)$, is the crossed product von Neumann algebra $M \rtimes_{\sigma \varphi} \mathbf{R}$. The natural inclusion $\pi_{\varphi}: M \rightarrow \mathrm{c}_{\varphi}(M)$ and the unitary representation $\lambda_{\varphi}: \mathbf{R} \rightarrow \mathrm{c}_{\varphi}(M)$ satisfy the covariance relation

$$
\lambda_{\varphi}(t) \pi_{\varphi}(x) \lambda_{\varphi}(t)^{*}=\pi_{\varphi}\left(\sigma_{t}^{\varphi}(x)\right) \quad \text { for all } x \in M \text { and all } t \in \mathbf{R} .
$$

Put $\mathrm{L}_{\varphi}(\mathbf{R})=\lambda_{\varphi}(\mathbf{R})^{\prime \prime}$. There is a unique faithful normal conditional expectation $\mathrm{E}_{\mathrm{L}_{\varphi}(\mathbf{R})}$ : $\mathrm{c}_{\varphi}(M) \rightarrow \mathrm{L}_{\varphi}(\mathbf{R})$ satisfying $\mathrm{E}_{\mathrm{L}_{\varphi}(\mathbf{R})}\left(\pi_{\varphi}(x) \lambda_{\varphi}(t)\right)=\varphi(x) \lambda_{\varphi}(t)$. The faithful normal semifinite weight defined by $f \mapsto \int_{\mathbf{R}} \exp (-s) f(s) \mathrm{d} s$ on $\mathrm{L}^{\infty}(\mathbf{R})$ gives rise to a faithful normal semifinite weight $\operatorname{Tr}_{\varphi}$ on $\mathrm{L}_{\varphi}(\mathbf{R})$ via the Fourier transform. The formula $\operatorname{Tr}_{\varphi}=\operatorname{Tr}_{\varphi} \circ \mathrm{E}_{\mathrm{L}_{\varphi}(\mathbf{R})}$ extends it to a faithful normal semifinite trace on $\mathrm{c}_{\varphi}(M)$.

Because of Connes's Radon-Nikodym cocycle theorem [Co72, Théorème 1.2.1] (see also [Ta03, Theorem VIII.3.3]), the semifinite von Neumann algebra $c_{\varphi}(M)$ together with its trace $\operatorname{Tr}_{\varphi}$ does not depend on the choice of $\varphi$ in the following precise sense. If $\psi$ is another faithful normal state on $M$, there is a canonical surjective $*$-isomorphism $\Pi_{\varphi, \psi}: \mathrm{c}_{\psi}(M) \rightarrow \mathrm{c}_{\varphi}(M)$ such that $\Pi_{\varphi, \psi} \circ \pi_{\psi}=\pi_{\varphi}$ and $\operatorname{Tr}_{\varphi} \circ \Pi_{\varphi, \psi}=\operatorname{Tr}_{\psi}$. Note however that $\Pi_{\varphi, \psi}$ does not map the subalgebra 
$\mathrm{L}_{\psi}(\mathbf{R}) \subset \mathrm{c}_{\psi}(M)$ onto the subalgebra $\mathrm{L}_{\varphi}(\mathbf{R}) \subset \mathrm{c}_{\varphi}(M)$ (and hence we use the symbol $\mathrm{L}_{\varphi}(\mathbf{R})$ instead of the usual $\mathrm{L}(\mathbf{R})$ ).

In order to prove the asymptotic orthogonality property inside arbitrary free product von Neumann algebras (see Theorem 3.1 below), we will use the following key simple lemma whose proof is similar to [MU12, Proposition 2.8] using [HS90, Theorem 11.1].

Lemma 2.1. Let $(M, \varphi)$ be any diffuse $\sigma$-finite von Neumann algebra endowed with a faithful normal state. For every $\delta>0$, there exists a faithful normal state $\psi \in M_{*}$ such that $\|\varphi-\psi\|<\delta$ and such that the centralizer $M^{\psi}$ is diffuse.

Proof. Write $M=M_{d} \oplus M_{c}$ where $M_{d}$ is of type I with diffuse center and $M_{c}$ has no type I direct summand. The above decomposition gives $\varphi=\varphi_{d}+\varphi_{c}$. By [HS90, Theorem 11.1] (which dates back to Connes-Størmer's transitivity theorem [CS78), one can find a faithful normal positive linear functional $\varphi_{c}^{\prime} \in\left(M_{c}\right)_{*}$ such that $\left\|\varphi_{c}^{\prime}\right\|_{\left(M_{c}\right)_{*}}=\left\|\varphi_{c}\right\|_{\left(M_{c}\right)_{*}},\left\|\varphi_{c}-\varphi_{c}^{\prime}\right\|_{\left(M_{c}\right)_{*}}<\delta$ and $\left(M_{c}\right)^{\varphi_{c}^{\prime}}$ is of type $\mathrm{II}_{1}$. Put $\psi:=\varphi_{d}+\varphi_{c}^{\prime}$ and observe that $\psi \in M_{*}$ is a faithful normal state. Then we have $\|\varphi-\psi\|_{M_{*}}=\left\|\varphi_{c}-\varphi_{c}^{\prime}\right\|_{\left(M_{c}\right)_{*}}<\delta$ and $\mathcal{Z}\left(M_{d}\right) \oplus\left(M_{c}\right)^{\varphi_{c}^{\prime}} \subset M^{\psi}$. Therefore, the centralizer $M^{\psi}$ is a diffuse von Neumann subalgebra (see e.g. [Bl06, Theorem IV.2.2.3]).

Popa's intertwining techniques. To fix notation, let $M$ be any $\sigma$-finite von Neumann algebra, $1_{A}$ and $1_{B}$ any nonzero projections in $M, A \subset 1_{A} M 1_{A}$ and $B \subset 1_{B} M 1_{B}$ any von Neumann subalgebras. Popa introduced his powerful intertwining-by-bimodules techniques in [Po01 in the case when $M$ is finite and more generally in [Po03] in the case when $M$ is endowed with an almost periodic faithful normal state $\varphi$ for which $1_{A} \in M^{\varphi}, A \subset 1_{A} M^{\varphi} 1_{A}$ and $1_{B} \in M^{\varphi}$, $B \subset 1_{B} M^{\varphi} 1_{B}$. It was showed in [HV12, Ue12] that Popa's intertwining techniques extend to the case when $B$ is finite and with expectation in $1_{B} M 1_{B}$ and $A \subset 1_{A} M 1_{A}$ is any von Neumann subalgebra.

In this paper, we will need the following generalization of [Po01, Theorem A.1] in the case when $A \subset 1_{A} M 1_{A}$ is any finite von Neumann subalgebra with expectation and $B \subset 1_{B} M 1_{B}$ is any von Neumann subalgebra with expectation.

Theorem 2.2 ([HI15, Theorem 4.3]). Let $M$ be any $\sigma$-finite von Neumann algebra, $1_{A}$ and $1_{B}$ any nonzero projections in $M, A \subset 1_{A} M 1_{A}$ and $B \subset 1_{B} M 1_{B}$ any von Neumann subalgebras with faithful normal conditional expectations $\mathrm{E}_{A}: 1_{A} M 1_{A} \rightarrow A$ and $\mathrm{E}_{B}: 1_{B} M 1_{B} \rightarrow B$ respectively. Assume moreover that $A$ is a finite von Neumann algebra.

Then the following conditions are equivalent:

(1) There exist projections $e \in A$ and $f \in B$, a nonzero partial isometry $v \in e M f$ and $a$ unital normal $*$-homomorphism $\theta: e A e \rightarrow f B f$ such that the inclusion $\theta(e A e) \subset f B f$ is with expectation and $a v=v \theta(a)$ for all $a \in e A e$.

(2) There exist $n \geq 1$, a projection $q \in \mathbf{M}_{n}(B)$, a nonzero partial isometry $v \in\left(1_{A} M \otimes\right.$ $\left.\mathbf{M}_{1, n}(\mathbf{C})\right) q$ and a unital normal *-homomorphism $\pi: A \rightarrow q \mathbf{M}_{n}(B) q$ such that the inclusion $\pi(A) \subset q \mathbf{M}_{n}(B) q$ is with expectation and av $=v \pi(a)$ for all $a \in A$.

(3) There exists no net $\left(w_{i}\right)_{i \in I}$ of unitaries in $\mathcal{U}(A)$ such that $\mathrm{E}_{B}\left(b^{*} w_{i} a\right) \rightarrow 0 \sigma$-strongly as $i \rightarrow \infty$ for all $a, b \in 1_{A} M 1_{B}$.

If one of the above conditions is satisfied, we will say that $A$ embeds with expectation into $B$ inside $M$ and write $A \preceq_{M} B$.

Moreover, HI15, Theorem 4.3] asserts that when $B \subset 1_{B} M 1_{B}$ is a semifinite von Neumann subalgebra endowed with any fixed faithful normal semifinite trace $\operatorname{Tr}$, then $A \preceq_{M} B$ if and only if there exist a projection $e \in A$, a Tr-finite projection $f \in B$, a nonzero partial isometry $v \in e M f$ and a unital normal $*$-homomorphism $\theta: e A e \rightarrow f B f$ such that $a v=v \theta(a)$ for all 
$a \in e A e$. Hence, in that case, the notation $A \preceq_{M} B$ is consistent with [Ue12, Proposition 3.1]. In particular, the projection $q \in \mathbf{M}_{n}(B)$ in Theorem 2.2 (2) is chosen to be finite under the trace $\operatorname{Tr} \otimes \operatorname{tr}_{n}$, when $B$ is semifinite with any fixed faithful normal semifinite trace $\operatorname{Tr}$. We refer to [HI15, Section 4] for further details.

We say that a $\sigma$-finite von Neumann algebra $P$ is tracial if it is endowed with a faithful normal tracial state $\tau$. Following Jo82, PP84, a unital inclusion of tracial von Neumann algebras $A \subset(P, \tau)$ has finite Jones index if $\operatorname{dim}_{A}\left(\mathrm{~L}^{2}(P, \tau)_{A}\right)<+\infty$ with the Murray-von Neumann dimension function $\operatorname{dim}_{A}$ determined by $\tau$. Following [Va07, Appendix A], a unital inclusion of tracial von Neumann algebras $A \subset(P, \tau)$ has essentially finite index if there exists a sequence of nonzero projections $\left(p_{n}\right)_{n}$ in $A^{\prime} \cap P$ such that the unital inclusion of tracial von Neumann algebras $A p_{n} \subset\left(p_{n} P p_{n}, \frac{\tau\left(p_{n} \cdot p_{n}\right)}{\tau\left(p_{n}\right)}\right)$ has finite Jones index for all $n \in \mathbf{N}$ and $p_{n} \rightarrow 1 \sigma$-strongly as $n \rightarrow \infty$.

We will need the following technical lemma about how the intertwining technique behaves with respect to taking subalgebras of essentially finite index.

Lemma 2.3 (Va07, Lemma 3.9]). Let $M$ be any $\sigma$-finite von Neumann algebra, $1_{P}$ and $1_{B}$ any nonzero projections in $M, P \subset 1_{P} M 1_{P}$ and $B \subset 1_{B} M 1_{B}$ any von Neumann subalgebras with expectation. Assume moreover that $P$ is a finite von Neumann algebra and $A \subset P$ is a unital von Neumann subalgebra of essentially finite index. Then $A \preceq_{M} B$ implies $P \preceq_{M} B$.

Proof. This result is [Va07, Lemma 3.9] when the ambient von Neumann algebra $M$ is finite and its proof applies mutatis mutandis to our more general setting.

We will moreover need the following two technical lemmas about intertwining subalgebras inside continuous cores.

Lemma 2.4. Let $(M, \varphi)$ be any $\sigma$-finite von Neumann algebra endowed with a faithful normal state. Let $q \in M^{\varphi}$ be any nonzero projection and $Q \subset q M q$ any von Neumann subalgebra that is globally invariant under the modular automorphism group $\sigma^{\varphi_{q}}$ of $\varphi_{q}=\frac{\varphi(q \cdot q)}{\varphi(q)}$. Denote by $\mathrm{E}_{Q}$ the unique $\varphi_{q}$-preserving conditional expectation from $q M q$ onto $Q$.

Then for every nonzero finite trace projection $p \in \mathrm{c}_{\varphi}(M)$ and every net $\left(u_{i}\right)_{i \in I}$ in $\operatorname{Ball}(M)$ such that $\lim _{i} \mathrm{E}_{Q}\left(b^{*} u_{i} a\right)=0 \sigma$-strongly for all $a, b \in M q$, we have

$$
\lim _{i}\left\|\mathrm{E}_{\mathrm{c}_{\varphi_{q}}(Q)}\left(y^{*} p \pi_{\varphi}\left(u_{i}\right) p x\right)\right\|_{2}=0, \forall x, y \in p \mathrm{c}_{\varphi}(M) \pi_{\varphi}(q) .
$$

In particular, for any faithful normal state $\psi \in M_{*}$, any nonzero projection $r \in M^{\psi}$, any von Neumann subalgebra $R \subset r M^{\psi} r$ satisfying $R \npreceq_{M} Q$ and any finite trace projection $s \in \mathrm{L}_{\psi}(\mathbf{R})$, we have

$$
\Pi_{\varphi, \psi}\left(\pi_{\psi}(R) s\right) \npreceq_{\mathrm{c}_{\varphi}(M)} \mathrm{c}_{\varphi_{q}}(Q) .
$$

Proof. The proof is essentially contained in [BHR12, Proposition 2.10] (see also [HR10, Proposition 5.3]). Simply denote by $\operatorname{Tr}=\operatorname{Tr}_{\varphi}$ the canonical trace on $c_{\varphi}(M)$ and by $\|\cdot\|_{2}$ the $\mathrm{L}^{2}$-norm with respect to Tr. Let $x, y \in \operatorname{Ball}\left(p \mathrm{c}_{\varphi}(M) \pi_{\varphi}(q)\right)$ be any elements. Fix an increasing sequence $\left(p_{m}\right)_{m}$ of finite trace projections in $\mathrm{L}_{\varphi}(\mathbf{R})$ such that $p_{m} \rightarrow 1 \sigma$-strongly. Observe that $p_{m} \pi_{\varphi}(q)=\pi_{\varphi}(q) p_{m}$ for all $m \in \mathbf{N}$, since $q \in M^{\varphi}$.

Let $\varepsilon>0$. Since $\operatorname{Tr}(p)<+\infty$, we may choose $m \in \mathbf{N}$ large enough such that

$$
\left\|p x-p x p_{m}\right\|_{2}+\left\|y^{*} p-p_{m} y^{*} p\right\|_{2}<\frac{\varepsilon}{2} .
$$

Observe that the unital $*$-subalgebra

$$
\mathcal{A}:=\left\{\sum_{j=1}^{n} \pi_{\varphi}\left(a_{j}\right) \lambda_{\varphi}\left(t_{j}\right): n \geq 1, a_{1}, \ldots, a_{n} \in M, t_{1}, \ldots, t_{n} \in \mathbf{R}\right\}
$$


is $\sigma$-*-strongly dense in $\mathrm{c}_{\varphi}(M)$. Using Kaplansky's density theorem and since $\operatorname{Tr}\left(p_{m}\right)<+\infty$, there exist $x_{0}, y_{0} \in \operatorname{Ball}\left(\mathcal{A} \pi_{\varphi}(q)\right)$ such that

$$
\left\|p x p_{m}-x_{0} p_{m}\right\|_{2}+\left\|p_{m} y^{*} p-p_{m} y_{0}^{*}\right\|_{2}<\frac{\varepsilon}{2} .
$$

Using (2.1) and (2.2), for all $i \in I$, we have

$$
\left\|\mathrm{E}_{\mathrm{c}_{\varphi_{q}}(Q)}\left(y^{*} p \pi_{\varphi}\left(u_{i}\right) p x\right)\right\|_{2} \leq\left\|\mathrm{E}_{\mathrm{C}_{\varphi_{q}}(Q)}\left(p_{m} y_{0}^{*} \pi_{\varphi}\left(u_{i}\right) x_{0} p_{m}\right)\right\|_{2}+\varepsilon .
$$

Write $x_{0}=\sum_{j=1}^{\ell} \pi_{\varphi}\left(a_{j}\right) \lambda_{\varphi}\left(t_{j}\right)$ and $y_{0}=\sum_{k=1}^{n} \pi_{\varphi}\left(b_{k}\right) \lambda_{\varphi}\left(t_{k}^{\prime}\right)$ for some $a_{j}, b_{k} \in M q$ and $t_{j}, t_{k}^{\prime} \in$ R. Since

$$
\mathrm{E}_{\mathrm{c}_{\varphi_{q}}(Q)}\left(p_{m} y_{0}^{*} \pi_{\varphi}\left(u_{i}\right) x_{0} p_{m}\right)=\sum_{j, k} p_{m} \lambda_{\varphi}\left(t_{k}^{\prime}\right)^{*} \pi_{\varphi}\left(\mathrm{E}_{Q}\left(b_{k}^{*} u_{i} a_{j}\right)\right) \lambda_{\varphi}\left(t_{j}\right) p_{m}
$$

and since $\lim _{i} \mathrm{E}_{Q}\left(b_{k}^{*} u_{i} a_{j}\right)=0 \sigma$-strongly for all $j, k$ and since $\operatorname{Tr}\left(p_{m}\right)<+\infty$, we obtain

$$
\lim _{i}\left\|\mathrm{E}_{\mathrm{C}_{\varphi_{q}}(Q)}\left(p_{m} y_{0}^{*} \pi_{\varphi}\left(u_{i}\right) x_{0} p_{m}\right)\right\|_{2}=0 .
$$

Then (2.3) and (2.4) imply that $\lim \sup _{i}\left\|\mathrm{E}_{\mathrm{c}_{\varphi_{q}}(Q)}\left(y^{*} p \pi_{\varphi}\left(u_{i}\right) p x\right)\right\|_{2} \leq \varepsilon$. Since $\varepsilon>0$ is arbitrary, we finally obtain

$$
\lim _{i}\left\|\mathrm{E}_{\mathrm{C}_{\varphi q}(Q)}\left(y^{*} p \pi_{\varphi}\left(u_{i}\right) p x\right)\right\|_{2}=0 .
$$

Next, assume that $\psi \in M_{*}$ is any faithful normal state, $r \in M^{\psi}$ is any nonzero projection, $R \subset r M^{\psi} r$ is any von Neumann subalgebra such that $R \npreceq_{M} Q$ and $s \in \mathrm{L}_{\psi}(\mathbf{R})$ is any nonzero finite trace projection. By Theorem [2.2, there exists a net $\left(v_{i}\right)_{j \in J}$ in $\mathcal{U}(R)$ such that $\lim _{j}\left\|\mathrm{E}_{Q}\left(b^{*} v_{j} a\right)\right\|_{\varphi}=0$ for all $a, b \in r M q$. Recall that $\Pi_{\varphi, \psi} \circ \pi_{\psi}=\pi_{\varphi}$ and $\operatorname{Tr}_{\varphi} \circ \Pi_{\varphi, \psi}=\operatorname{Tr}_{\psi}$. Put $p=\Pi_{\varphi, \psi}(s)$. The first part of the proof implies that $\lim _{j}\left\|\mathrm{E}_{\mathrm{C}_{\varphi_{q}}(Q)}\left(y^{*} \pi_{\varphi}\left(v_{j}\right) p x\right)\right\|_{2}=0$ for all $x, y \in p \pi_{\varphi}(r) \mathrm{c}_{\varphi}(M) \pi_{\varphi}(q)$. Since $\pi_{\varphi}\left(v_{j}\right) p=\Pi_{\varphi, \psi}\left(\pi_{\psi}\left(v_{j}\right) s\right) \in \mathcal{U}\left(\Pi_{\varphi, \psi}\left(\pi_{\psi}(R) s\right)\right)$ for all $j \in J$, we obtain that $\Pi_{\varphi, \psi}\left(\pi_{\psi}(R) s\right) \npreceq_{\mathrm{c}_{\varphi}(M)} \mathrm{c}_{\varphi_{q}}(Q)$ by Theorem 2.2 .

Lemma 2.5. Let $M$ be any $\sigma$-finite von Neumann algebra and $\varphi, \psi \in M_{*}$ any faithful states. Let $q \in M^{\psi}$ be any nonzero projection and $Q \subset q M q$ any diffuse von Neumann subalgebra that is globally invariant under the modular automorphism group $\sigma^{\psi_{q}}$ of $\psi_{q}=\frac{\psi(q \cdot q)}{\psi(q)}$. Then for every nonzero finite trace projection $p \in \mathrm{L}_{\psi}(\mathbf{R})$, we have $c_{\psi_{q}}(Q) \subset c_{\psi}(M)$ naturally and

$$
\Pi_{\varphi, \psi}\left(p \mathrm{c}_{\psi_{q}}(Q) p\right) \npreceq_{\mathrm{c}_{\varphi}(M)} \mathrm{L}_{\varphi}(\mathbf{R}) .
$$

Proof. Denote by $z \in \mathcal{Z}(Q)$ the unique central projection such that $Q z$ is of type I and $Q z^{\perp}$ has no type I direct summand. Observe that $z \in M^{\psi}, \pi_{\psi}(z) \in \mathcal{Z}\left(\mathrm{c}_{\psi_{q}}(Q)\right)$ and

$$
p \mathrm{c}_{\psi_{q}}(Q) p=p \mathrm{c}_{\psi_{q}}(Q) p \pi_{\psi}(z) \oplus p \mathrm{c}_{\psi_{q}}(Q) p \pi_{\psi}\left(z^{\perp}\right)=p \mathrm{c}_{\psi_{z}}(Q z) p \oplus p \mathrm{c}_{\psi_{z} \perp}\left(Q z^{\perp}\right) p .
$$

Since $Q z^{\perp}$ has no type I direct summand, $p \mathrm{c}_{\psi_{z \perp}}\left(Q z^{\perp}\right) p$ has no type I direct summand either. This follows from the fact that continuous cores are independent of states or even weights (due to Connes's Radon-Nykodym cocycle theorem Co72, Théorème 1.2.1]) as well as the fact that the continuous core of any type III von Neumann algebra must be of type $\mathrm{II}_{\infty}$ (see Ta03, Theorem XII.1.1]). Hence we have

$$
\Pi_{\varphi, \psi}\left(p \mathrm{c}_{\psi_{q}}(Q) p \pi_{\psi}\left(z^{\perp}\right)\right) \npreceq_{\mathrm{c}_{\varphi}(M)} \mathrm{L}_{\varphi}(\mathbf{R}) .
$$

Since $Q z$ is of type I and diffuse, $\mathcal{Z}(Q z) \subset Q^{\psi_{q}} z=(Q z)^{\psi_{z}}$ with $\psi_{z}:=\frac{\psi(z \cdot z)}{\psi(z)}$ is also diffuse and hence $\mathcal{Z}(Q z) \npreceq{ }_{M} \mathbf{C} 1$. Then Lemma 2.4 (with letting the $Q$ there be the trivial algebra) implies that

$$
\Pi_{\varphi, \psi}\left(p c_{\psi_{q}}(Q) p \pi_{\psi}(z)\right) \npreceq_{\mathrm{c}_{\varphi}(M)} \mathrm{L}_{\varphi}(\mathbf{R}) .
$$

Combining the above two facts, we finally obtain that $\Pi_{\varphi, \psi}\left(p \mathrm{c}_{\psi_{q}}(Q) p\right) \npreceq_{\mathrm{c}_{\varphi}(M)} \mathrm{L}_{\varphi}(\mathbf{R})$. 
Amalgamated free product von Neumann algebras. For each $i \in\{1,2\}$, let $B \subset M_{i}$ be any inclusion of $\sigma$-finite von Neumann algebras with faithful normal conditional expectation $\mathrm{E}_{i}: M_{i} \rightarrow B$. The amalgamated free product $(M, \mathrm{E})=\left(M_{1}, \mathrm{E}_{1}\right) *_{B}\left(M_{2}, \mathrm{E}_{2}\right)$ is a pair of von Neumann algebra $M$ generated by $M_{1}$ and $M_{2}$ and faithful normal conditional expectation $\mathrm{E}: M \rightarrow B$ such that $M_{1}, M_{2}$ are freely independent with respect to $\mathrm{E}$ :

$$
\mathrm{E}\left(x_{1} \cdots x_{n}\right)=0 \text { whenever } x_{j} \in M_{i_{j}}^{\circ} \text { and } i_{1} \neq \cdots \neq i_{n} \text {. }
$$

Here and in what follows, we denote by $M_{i}^{\circ}=\operatorname{ker}\left(\mathrm{E}_{i}\right)$. We refer to the product $x_{1} \cdots x_{n}$ where $x_{j} \in M_{i_{j}}^{\circ}$ and $i_{1} \neq \cdots \neq i_{n}$ as a reduced word in $M_{i_{1}}^{\circ} \cdots M_{i_{n}}^{\circ}$ of length $n \geq 1$. The linear span of $B$ and of all the reduced words in $M_{i_{1}}^{\circ} \cdots M_{i_{n}}^{\circ}$ where $n \geq 1$ and $i_{1} \neq \cdots \neq i_{n}$ forms a unital $\sigma$-strongly dense $*$-subalgebra of $M$. We call the resulting $M$ the amalgamated free product von Neumann algebra of $\left(M_{1}, \mathrm{E}_{1}\right)$ and $\left(M_{2}, \mathrm{E}_{2}\right)$.

When $B=\mathbf{C} 1, \mathrm{E}_{i}=\varphi_{i}(\cdot) 1$ for all $i \in\{1,2\}$ and $\mathrm{E}=\varphi(\cdot) 1$, we will simply denote by $(M, \varphi)=\left(M_{1}, \varphi_{1}\right) *\left(M_{2}, \varphi_{2}\right)$ and call the resulting $M$ the free product von Neumann algebra of $\left(M_{1} \varphi_{1}\right)$ and $\left(M_{2}, \varphi_{2}\right)$.

When $B$ is a semifinite von Neumann algebra with faithful normal semifinite trace $\operatorname{Tr}$ and the weight $\operatorname{Tr} \circ \mathrm{E}_{i}$ is tracial on $M_{i}$ for every $i \in\{1,2\}$, then the weight $\operatorname{Tr} \circ \mathrm{E}$ is tracial on $M$ (see [Po90, Proposition 3.1] for the finite case and [Ue98a, Theorem 2.6] for the general case). In particular, $M$ is a semifinite von Neumann algebra. In that case, we will refer to $(M, \mathrm{E})=\left(M_{1}, \mathrm{E}_{1}\right) *_{B}\left(M_{2}, \mathrm{E}_{2}\right)$ as a semifinite amalgamated free product.

Let $\varphi \in B_{*}$ be any faithful normal state. Then for all $t \in \mathbf{R}$, we have $\sigma_{t}^{\varphi \circ \mathrm{E}}=\sigma_{t}^{\varphi \circ \mathrm{E}_{1}} * \sigma_{t}^{\varphi \circ \mathrm{E}_{2}}$ (see [Ue98a, Theorem 2.6]). By [Ta03, Theorem IX.4.2], there exists a unique $\varphi \circ$ E-preserving conditional expectation $\mathrm{E}_{M_{1}}: M \rightarrow M_{1}$. Moreover, we have $\mathrm{E}_{M_{1}}\left(x_{1} \cdots x_{n}\right)=0$ for all the reduced words $x_{1} \cdots x_{n}$ that contain at least one letter from $M_{2}^{\circ}$ (see e.g. [Ue10, Lemma 2.1]). We will denote by $M \ominus M_{1}=\operatorname{ker}\left(\mathrm{E}_{M_{1}}\right)$. For more on (amalgamated) free product von Neumann algebras, we refer the reader to [BHR12, Po90, Ue98a, Ue10, Ue12, Vo85, VDN92,

Lemma 2.6. For each $i \in\{1,2\}$, let $B \subset M_{i}$ be any inclusion of $\sigma$-finite von Neumann algebras with faithful normal conditional expectations $\mathrm{E}_{i}: M_{i} \rightarrow B$. Denote by $(M, \mathrm{E})=$ $\left(M_{1}, \mathrm{E}_{1}\right) *_{B}\left(M_{2}, \mathrm{E}_{2}\right)$ the amalgamated free product.

Let $\psi \in M_{*}$ be any faithful normal state such that $\psi=\psi \circ \mathrm{E}_{M_{1}}$. Let $\left(u_{j}\right)_{j \in J}$ be any net in $\operatorname{Ball}\left(\left(M_{1}\right)^{\psi}\right)$ such that $\lim _{j} \mathrm{E}_{1}\left(b^{*} u_{j} a\right)=0$-strongly for all $a, b \in M_{1}$. Then for all $x, y \in$ $M \ominus M_{1}$, we have that $\lim _{j} \mathrm{E}_{M_{1}}\left(y^{*} u_{j} x\right)=0 \sigma$-strongly.

Proof. We first prove the $\sigma$-strong convergence when $x, y \in M_{1} M_{2}^{\circ} \cdots M_{2}^{\circ} M_{1}$ are words of the form $x=a x^{\prime} c$ and $y=b y^{\prime} d$ with $a, b, c, d \in M_{1}$ and $x^{\prime}, y^{\prime} \in M_{2}^{\circ} \cdots M_{2}^{\circ}$. By freeness with amalgamation over $B$, for all $n \in \mathbf{N}$, we have

$$
\mathrm{E}_{M_{1}}\left(y^{*} u_{j} x\right)=\mathrm{E}_{M_{1}}\left(d^{*} y^{\prime *} b^{*} u_{j} a x^{\prime} c\right)=\mathrm{E}_{M_{1}}\left(d^{*} y^{\prime *} \mathrm{E}_{1}\left(b^{*} u_{j} a\right) x^{\prime} c\right) .
$$

Since $\lim _{j} \mathrm{E}_{1}\left(b^{*} u_{j} a\right)=0 \sigma$-strongly, we have $\lim _{j} \mathrm{E}_{M_{1}}\left(y^{*} u_{j} x\right)=0 \sigma$-strongly.

Recall that $\psi=\psi \circ \mathrm{E}_{M_{1}}$. We next prove the $\sigma$-strong convergence when $x \in M \ominus M_{1}$ is any element and $y \in M_{1} M_{2}^{\circ} \cdots M_{2}^{\circ} M_{1}$ is any word as above. Indeed, we may choose a sequence $\left(x_{k}\right)_{k}$, where each $x_{k}$ is a finite linear combination of words in $M_{1} M_{2}^{\circ} \cdots M_{2}^{\circ} M_{1}$, and such that $\lim _{k \rightarrow \infty}\left\|x-x_{k}\right\|_{\psi}=0$. Then by triangle inequality, for all $j \in J$ and $k \in \mathbf{N}$, we have

$$
\begin{aligned}
\left\|\mathrm{E}_{M_{1}}\left(y^{*} u_{j} x\right)\right\|_{\psi} & \leq\left\|\mathrm{E}_{M_{1}}\left(y^{*} u_{j} x_{k}\right)\right\|_{\psi}+\left\|\mathrm{E}_{M_{1}}\left(y^{*} u_{j}\left(x-x_{k}\right)\right)\right\|_{\psi} \\
& \leq\left\|\mathrm{E}_{M_{1}}\left(y^{*} u_{j} x_{k}\right)\right\|_{\psi}+\left\|y^{*} u_{n}\left(x-x_{k}\right)\right\|_{\psi} \\
& \leq\left\|\mathrm{E}_{M_{1}}\left(y^{*} u_{j} x_{k}\right)\right\|_{\psi}+\|y\|_{\infty}\left\|x-x_{k}\right\|_{\psi} .
\end{aligned}
$$

The first part of the proof implies that $\lim \sup _{j}\left\|\mathrm{E}_{M_{1}}\left(y^{*} u_{j} x\right)\right\|_{\psi} \leq\|y\|_{\infty}\left\|x-x_{k}\right\|_{\psi}$ for all $k \in \mathbf{N}$ and hence $\lim _{j}\left\|\mathrm{E}_{M_{1}}\left(y^{*} u_{j} x\right)\right\|_{\psi}=0$. 
Recall that $\psi=\psi \circ \mathrm{E}_{M_{1}}$ and hence $\sigma_{t}^{\psi}\left(M_{1}\right)=M_{1}$ for all $t \in \mathbf{R}$. We next prove the $\sigma$ strong convergence when $x \in M \ominus M_{1}$ is any analytic element with respect to the modular automorphism group $\sigma^{\psi}$ and $y \in M \ominus M_{1}$ is any element. Indeed, we may choose a sequence $\left(y_{k}\right)_{k}$, where each $y_{k}$ is a finite linear combination of words in $M_{1} M_{2}^{\circ} \cdots M_{2}^{\circ} M_{1}$, and such that $\lim _{k \rightarrow \infty}\left\|y^{*}-y_{k}^{*}\right\|_{\psi}=0$. Then by triangle inequality, for all $j \in J$ and all $k \in \mathbf{N}$, we have

$$
\begin{aligned}
\left\|\mathrm{E}_{M_{1}}\left(y^{*} u_{j} x\right)\right\|_{\psi} & \leq\left\|\mathrm{E}_{M_{1}}\left(y_{k}^{*} u_{j} x\right)\right\|_{\psi}+\left\|\mathrm{E}_{M_{1}}\left(\left(y^{*}-y_{k}^{*}\right) u_{n} x\right)\right\|_{\psi} \\
& \leq\left\|\mathrm{E}_{M_{1}}\left(y_{k}^{*} u_{j} x\right)\right\|_{\psi}+\left\|\left(y^{*}-y_{k}^{*}\right) u_{j} x\right\|_{\psi} \\
& =\left\|\mathrm{E}_{M_{1}}\left(y_{k}^{*} u_{j} x\right)\right\|_{\psi}+\left\|J^{M} \sigma_{\mathrm{i} / 2}^{\psi}(x)^{*} u_{j}^{*} J^{M}\left(y^{*}-y_{k}^{*}\right)\right\|_{\psi} \\
& =\left\|\mathrm{E}_{M_{1}}\left(y_{k}^{*} u_{j} x\right)\right\|_{\psi}+\left\|\sigma_{\mathrm{i} / 2}^{\psi}(x)\right\|_{\infty}\left\|y^{*}-y_{k}^{*}\right\|_{\psi} .
\end{aligned}
$$

The second part of the proof implies that $\lim \sup _{j}\left\|\mathrm{E}_{M_{1}}\left(y^{*} u_{j} x\right)\right\|_{\psi} \leq\left\|\sigma_{\mathrm{i} / 2}^{\psi}(x)\right\|_{\infty}\left\|y^{*}-y_{k}^{*}\right\|_{\psi}$ for all $k \in \mathbf{N}$ and hence $\lim _{j}\left\|\mathrm{E}_{M_{1}}\left(y^{*} u_{j} x\right)\right\|_{\psi}=0$.

We finally prove the $\sigma$-strong convergence when $x, y \in M \ominus M_{1}$ are any elements. Indeed, we may choose a sequence $\left(x_{k}\right)_{k}$ in $M \ominus M_{1}$ of analytic elements with respect to the modular automorphism group $\sigma^{\psi}$ such that $\lim _{k \rightarrow \infty}\left\|x-x_{k}\right\|_{\psi}=0$. Then by triangle inequality, for all $j \in J$ and all $k \in \mathbf{N}$, we have

$$
\begin{aligned}
\left\|\mathrm{E}_{M_{1}}\left(y^{*} u_{j} x\right)\right\|_{\psi} & \leq\left\|\mathrm{E}_{M_{1}}\left(y^{*} u_{j} x_{k}\right)\right\|_{\psi}+\left\|\mathrm{E}_{M_{1}}\left(y^{*} u_{j}\left(x-x_{k}\right)\right)\right\|_{\psi} \\
& \leq\left\|\mathrm{E}_{M_{1}}\left(y^{*} u_{j} x_{k}\right)\right\|_{\psi}+\left\|y^{*} u_{j}\left(x-x_{k}\right)\right\|_{\psi} \\
& \leq\left\|\mathrm{E}_{M_{1}}\left(y^{*} u_{j} x_{k}\right)\right\|_{\psi}+\|y\|_{\infty}\left\|x-x_{k}\right\|_{\psi} .
\end{aligned}
$$

The third part of the proof implies that $\lim \sup _{j}\left\|\mathrm{E}_{M_{1}}\left(y^{*} u_{j} x\right)\right\|_{\psi} \leq\|y\|_{\infty}\left\|x-x_{k}\right\|_{\psi}$ for all $k \in \mathbf{N}$ and hence $\lim _{j}\left\|\mathrm{E}_{M_{1}}\left(y^{*} u_{j} x\right)\right\|_{\psi}=0$. This finishes the proof of Lemma 2.6.

The next proposition about controlling the (quasi)-normalizer of diffuse subalgebras inside free product von Neumann algebras will be very useful in the proof of Theorem $\mathrm{A}$. This is a variant of [IPP05, Theorem 1.1] and [Ue12, Proposition 3.3], but the proof uses an idea of Va06, Lemma D3] and the previous lemma crucially. We point out that the first assertion also generalizes [Ue10, Corollary 3.2] (with $n=1, \pi(x)=u x u^{*}$ and $v=u$ for $u \in \mathcal{U}\left(A^{\prime} \cap M\right)$ or $\left.\mathcal{N}_{M}(A)\right)$. A more general, unified statement seems possible in the framework of amalgamated free products because the previous lemma is quite general, but the statements below fit the later use.

Proposition 2.7. For each $i \in\{1,2\}$, let $\left(M_{i}, \varphi_{i}\right)$ be any $\sigma$-finite von Neumann algebra endowed with a faithful normal state. Denote by $(M, \varphi)=\left(M_{1}, \varphi_{1}\right) *\left(M_{2}, \varphi_{2}\right)$ the free product.

(1) Let $1_{Q} \in M_{1}$ be any nonzero projection and $Q \subset 1_{Q} M_{1} 1_{Q}$ any diffuse von Neumann subalgebra with expectation. For every $n \geq 1$, every (not necessarily unital) normal *-homomorphism $\pi: Q \rightarrow \mathbf{M}_{n}\left(M_{1}\right)$ and every nonzero partial isometry $v \in\left(1_{Q} M \otimes\right.$ $\left.\mathbf{M}_{1, n}(\mathbf{C})\right) \pi\left(1_{Q}\right)$ such that $x v=v \pi(x)$ for all $x \in Q$, we have

$$
v \in\left(1_{Q} M_{1} \otimes \mathbf{M}_{1, n}(\mathbf{C})\right) \pi\left(1_{Q}\right) .
$$

(2) Let $1_{A} \in M$ be any nonzero projection and $A \subset 1_{A} M 1_{A}$ any diffuse von Neumann subalgebra with expectation. For every $n \geq 1$, every (not necessarily unital) normal $*$ homomorphism $\pi: A \rightarrow \mathbf{M}_{n}\left(M_{1}\right)$ such that the inclusion $\pi(A) \subset \pi\left(1_{A}\right) \mathbf{M}_{n}\left(M_{1}\right) \pi\left(1_{A}\right)$ is with expectation and every nonzero partial isometry $v \in\left(1_{A} M \otimes \mathbf{M}_{1, n}(\mathbf{C})\right) \pi\left(1_{A}\right)$ such that $a v=v \pi(a)$ for all $a \in A$, we have

$$
v^{*} v \in \pi\left(1_{A}\right) \mathbf{M}_{n}\left(M_{1}\right) \pi\left(1_{A}\right) \quad \text { and } \quad v^{*} \mathcal{N}_{1_{A} M 1_{A}}(A)^{\prime \prime} v \subset v^{*} v \mathbf{M}_{n}\left(M_{1}\right) v^{*} v .
$$

Proof. (1) As in the proof of Lemma 2.1 and since $Q \subset 1_{Q} M_{1} 1_{Q}$ is with expectation, we may choose a faithful normal state $\psi \in M_{*}$ such that $\psi=\psi \circ \mathrm{E}_{M_{1}}, 1_{Q} \in\left(M_{1}\right)^{\psi}, Q \subset 1_{Q} M 1_{Q}$ 
is globally invariant under the modular automorphism group $\sigma^{\psi_{Q}}$ and $Q^{\psi_{Q}} \subset 1_{Q}\left(M_{1}\right)^{\psi} 1_{Q}$ is diffuse where $\psi_{Q}:=\frac{\psi\left(1_{Q} \cdot 1_{Q}\right)}{\psi\left(1_{Q}\right)}$.

Let $n, \pi, v$ as in the statement. Denote by $\operatorname{tr}_{n}$ the canonical normalized trace on $\mathbf{M}_{n}(\mathbf{C})$ and write $v=\left[v_{1} \cdots v_{n}\right] \in\left(1_{Q} M \otimes \mathbf{M}_{1, n}(\mathbf{C})\right) \pi\left(1_{Q}\right)$. For all $x \in Q$, since $x v=v \pi(x)$, we have

$$
x \mathrm{E}_{\mathbf{M}_{n}\left(M_{1}\right)}(v)=\mathrm{E}_{\mathbf{M}_{n}\left(M_{1}\right)}(x v)=\mathrm{E}_{\mathbf{M}_{n}\left(M_{1}\right)}(v \pi(x))=\mathrm{E}_{\mathbf{M}_{n}\left(M_{1}\right)}(v) \pi(x)
$$

and hence

$$
x\left(v-\mathrm{E}_{\mathbf{M}_{n}\left(M_{1}\right)}(v)\right)=\left(v-\mathrm{E}_{\mathbf{M}_{n}\left(M_{1}\right)}(v)\right) \pi(x) .
$$

Put $w:=v-\mathrm{E}_{\mathbf{M}_{n}\left(M_{1}\right)}(v) \in\left(1_{Q}\left(M \ominus M_{1}\right) \otimes \mathbf{M}_{1, n}(\mathbf{C})\right) \pi\left(1_{Q}\right)$ and write $w=\left[w_{1} \cdots w_{n}\right]$ with $w_{1}, \ldots, w_{n} \in 1_{Q}\left(M \ominus M_{1}\right)$. Fix a sequence of unitaries $\left(u_{k}\right)_{k}$ in $\mathcal{U}\left(Q^{\psi_{Q}}\right)$ such that $\lim _{k \rightarrow \infty} u_{k}=0$ $\sigma$-weakly. By Lemma 2.6, we have

$$
\lim _{k \rightarrow \infty}\left\|\mathrm{E}_{\mathbf{M}_{n}\left(M_{1}\right)}\left(w^{*} u_{k} w\right)\right\|_{\psi \otimes \operatorname{tr}_{n}}^{2}=\lim _{k \rightarrow \infty} \sum_{i, j=1}^{n}\left\|\mathrm{E}_{M_{1}}\left(w_{i}^{*} u_{k} w_{j}\right)\right\|_{\psi}^{2}=0 .
$$

Using (2.5) and (2.6) and since $\pi\left(u_{k}\right) \in \mathcal{U}(\pi(Q))$ and $w^{*} w \in \pi(Q)^{\prime} \cap \pi\left(1_{Q}\right) \mathbf{M}_{n}(M) \pi\left(1_{Q}\right)$, we have

$$
\begin{aligned}
\left\|\mathrm{E}_{\mathbf{M}_{n}\left(M_{1}\right)}\left(w^{*} w\right)\right\|_{\psi \otimes \operatorname{tr}_{n}} & =\limsup _{k \rightarrow \infty}\left\|\pi\left(u_{k}\right) \mathrm{E}_{\mathbf{M}_{n}\left(M_{1}\right)}\left(w^{*} w\right)\right\|_{\psi \otimes \operatorname{tr}_{n}} \\
& =\limsup _{k \rightarrow \infty}\left\|\mathrm{E}_{\mathbf{M}_{n}\left(M_{1}\right)}\left(\pi\left(u_{k}\right) w^{*} w\right)\right\|_{\psi \otimes \operatorname{tr}_{n}} \\
& =\limsup _{k \rightarrow \infty}\left\|\mathrm{E}_{\mathbf{M}_{n}\left(M_{1}\right)}\left(w^{*} w \pi\left(u_{k}\right)\right)\right\|_{\psi \otimes \operatorname{tr}_{n}} \\
& =\lim _{k \rightarrow \infty}\left\|\mathrm{E}_{\mathbf{M}_{n}\left(M_{1}\right)}\left(w^{*} u_{k} w\right)\right\|_{\psi \otimes \operatorname{tr}_{n}} \\
& =0 .
\end{aligned}
$$

This implies that $w^{*} w=0$ and hence $w=0$. Thus $v=\mathrm{E}_{\mathbf{M}_{n}\left(M_{1}\right)}(v) \in\left(1_{Q} M_{1} \otimes \mathbf{M}_{1, n}(\mathbf{C})\right) \pi\left(1_{Q}\right)$.

(2) We will be working inside the amalgamated free product von Neumann algebra

$$
\mathbf{M}_{n}(M)=\left(\mathbf{M}_{n}\left(M_{1}\right), \varphi_{1} \otimes \mathrm{id}_{n}\right) * \mathbf{M}_{n}(\mathbf{C})\left(\mathbf{M}_{n}\left(M_{2}\right), \varphi_{2} \otimes \mathrm{id}_{n}\right),
$$

and substitute formula (2.7) below for the assumption of item (1) that $x v=v \pi(x)$ for all $x \in Q$. Since $\pi(A) \subset \pi\left(1_{A}\right) \mathbf{M}_{n}\left(M_{1}\right) \pi\left(1_{A}\right)$ is a diffuse von Neumann subalgebra with expectation, we may choose, as in the proof of item (1), a faithful normal state $\psi \in \mathbf{M}_{n}(M)_{*}$ such that $\psi=\psi \circ \mathrm{E}_{\mathbf{M}_{n}\left(M_{1}\right)}, \pi\left(1_{A}\right) \in \mathbf{M}_{n}\left(M_{1}\right)^{\psi}$ and $\pi(A) \cap \pi\left(1_{A}\right) \mathbf{M}_{n}\left(M_{1}\right)^{\psi} \pi\left(1_{A}\right)$ is diffuse. Fix a sequence of unitaries $\left(u_{k}\right)_{k}$ in $\pi(A) \cap \pi\left(1_{A}\right) \mathbf{M}_{n}\left(M_{1}\right)^{\psi} \pi\left(1_{A}\right)$ such that $\lim _{k \rightarrow \infty} u_{k}=0 \sigma$-weakly. For each $k \in \mathbf{N}$, we may write $u_{k}=\pi\left(a_{k}\right)$ with a unitary $a_{k} \in A$.

Let now $x \in \mathcal{N}_{1_{A} M 1_{A}}(A)$ be any normalizing unitary element. Then for all $a \in A$, we have

$$
v^{*} x v \pi(a)=v^{*} x a v=v^{*}\left(x a x^{*}\right) x v=\pi\left(x a x^{*}\right) v^{*} x v,
$$

and hence, as in the proof of item (1), for every $k \in \mathbf{N}$ we have

$$
\left(v^{*} x v-\mathrm{E}_{\mathbf{M}_{n}\left(M_{1}\right)}\left(v^{*} x v\right)\right) u_{k}=\pi\left(x a_{k} x^{*}\right)\left(v^{*} x v-\mathrm{E}_{\mathbf{M}_{n}\left(M_{1}\right)}\left(v^{*} x v\right)\right) .
$$

Put $w:=v^{*} x v-\mathrm{E}_{\mathbf{M}_{n}\left(M_{1}\right)}\left(v^{*} x v\right) \in \pi\left(1_{A}\right)\left(\mathbf{M}_{n}(M) \ominus \mathbf{M}_{n}\left(M_{1}\right)\right) \pi\left(1_{A}\right)$. Using (2.8) and Lemma 2.6 and since $\pi\left(x a_{k} x^{*}\right) \in \mathcal{U}(\pi(A))$, we obtain, as in the proof of item (1), that

$$
\left\|\mathrm{E}_{\mathbf{M}_{n}\left(M_{1}\right)}\left(w w^{*}\right)\right\|_{\psi}=\lim _{k \rightarrow \infty}\left\|\mathrm{E}_{\mathbf{M}_{n}\left(M_{1}\right)}\left(w u_{k} w^{*}\right)\right\|_{\psi}=0
$$

implying that $v^{*} x v=\mathrm{E}_{\mathbf{M}_{n}\left(M_{1}\right)}\left(v^{*} x v\right) \in \mathbf{M}_{n}\left(M_{1}\right)$ and the desired assertion is immediate. 
Ultraproduct von Neumann algebras. Let $M$ be any $\sigma$-finite von Neumann algebra. Define

$$
\begin{aligned}
\mathcal{I}_{\omega}(M) & =\left\{\left(x_{n}\right)_{n} \in \ell^{\infty}(\mathbf{N}, M): x_{n} \rightarrow 0 * \text {-strongly as } n \rightarrow \omega\right\}, \\
\mathcal{M}^{\omega}(M) & =\left\{\left(x_{n}\right)_{n} \in \ell^{\infty}(\mathbf{N}, M):\left(x_{n}\right)_{n} \mathcal{I}_{\omega}(M) \subset \mathcal{I}_{\omega}(M) \text { and } \mathcal{I}_{\omega}(M)\left(x_{n}\right)_{n} \subset \mathcal{I}_{\omega}(M)\right\} .
\end{aligned}
$$

We have that the multiplier algebra $\mathcal{M}^{\omega}(M)$ is a $\mathrm{C}^{*}$-algebra and $\mathcal{I}_{\omega}(M) \subset \mathcal{M}^{\omega}(M)$ is a norm closed two-sided ideal. Following Oc85, we define the ultraproduct von Neumann algebra $M^{\omega}$ by $M^{\omega}=\mathcal{M}^{\omega}(M) / \mathcal{I}_{\omega}(M)$. We denote the image of $\left(x_{n}\right)_{n} \in \mathcal{M}^{\omega}(M)$ by $\left(x_{n}\right)^{\omega} \in M^{\omega}$.

For all $x \in M$, the constant sequence $(x)_{n}$ lies in the multiplier algebra $\mathcal{M}^{\omega}(M)$. We will then identify $M$ with $\left(M+\mathcal{I}_{\omega}(M)\right) / \mathcal{I}_{\omega}(M)$ and regard $M \subset M^{\omega}$ as a von Neumann subalgebra. The map $\mathrm{E}_{\omega}: M^{\omega} \rightarrow M:\left(x_{n}\right)^{\omega} \mapsto \sigma$-weak $\lim _{n \rightarrow \omega} x_{n}$ is a faithful normal conditional expectation. For every faithful normal state $\varphi \in M_{*}$, the formula $\varphi^{\omega}=\varphi \circ \mathrm{E}_{\omega}$ defines a faithful normal state on $M^{\omega}$. Observe that $\varphi^{\omega}\left(\left(x_{n}\right)^{\omega}\right)=\lim _{n \rightarrow \omega} \varphi\left(x_{n}\right)$ for all $\left(x_{n}\right)^{\omega} \in M^{\omega}$.

Let $Q \subset M$ be any von Neumann subalgebra with faithful normal conditional expectation $\mathrm{E}_{Q}: M \rightarrow Q$. Choose a faithful normal state $\varphi \in M_{*}$ such that $\varphi=\varphi \circ \mathrm{E}_{Q}$. We have $\ell^{\infty}(\mathbf{N}, Q) \subset \ell^{\infty}(\mathbf{N}, M), \mathcal{I}_{\omega}(Q) \subset \mathcal{I}_{\omega}(M)$ and $\mathcal{M}^{\omega}(Q) \subset \mathcal{M}^{\omega}(M)$. We will then identify $Q^{\omega}=\mathcal{M}^{\omega}(Q) / \mathcal{I}_{\omega}(Q)$ with $\left(\mathcal{M}^{\omega}(Q)+\mathcal{I}_{\omega}(M)\right) / \mathcal{I}_{\omega}(M)$ and regard $Q^{\omega} \subset M^{\omega}$ as a von Neumann subalgebra. Observe that the norm $\|\cdot\|_{\left(\left.\varphi\right|_{Q}\right)}$ on $Q^{\omega}$ is the restriction of the norm $\|\cdot\|_{\varphi^{\omega}}$ to $Q^{\omega}$. Observe moreover that $\left(\mathrm{E}_{Q}\left(x_{n}\right)\right)_{n} \in \mathcal{I}_{\omega}(Q)$ for all $\left(x_{n}\right)_{n} \in \mathcal{I}_{\omega}(M)$ and $\left(\mathrm{E}_{Q}\left(x_{n}\right)\right)_{n} \in \mathcal{M}^{\omega}(Q)$ for all $\left(x_{n}\right)_{n} \in \mathcal{M}^{\omega}(M)$. Therefore, the mapping $\mathrm{E}_{Q^{\omega}}: M^{\omega} \rightarrow Q^{\omega}:\left(x_{n}\right)^{\omega} \mapsto\left(\mathrm{E}_{Q}\left(x_{n}\right)\right)^{\omega}$ is a well-defined conditional expectation satisfying $\varphi^{\omega} \circ \mathrm{E}_{Q^{\omega}}=\varphi^{\omega}$. Hence, $\mathrm{E}_{Q^{\omega}}: M^{\omega} \rightarrow Q^{\omega}$ is a faithful normal conditional expectation.

Put $\mathcal{H}=\mathrm{L}^{2}(M)$. The ultraproduct Hilbert space $\mathcal{H}^{\omega}$ is defined to be the quotient of $\ell^{\infty}(\mathbf{N}, \mathcal{H})$ by the subspace consisting in sequences $\left(\xi_{n}\right)_{n}$ satisfying $\lim _{n \rightarrow \omega}\left\|\xi_{n}\right\|_{\mathcal{H}}=0$. We denote the image of $\left(\xi_{n}\right)_{n} \in \ell^{\infty}(\mathbf{N}, \mathcal{H})$ by $\left(\xi_{n}\right)_{\omega} \in \mathcal{H}^{\omega}$. The inner product space structure on the Hilbert space $\mathcal{H}^{\omega}$ is defined by $\left\langle\left(\xi_{n}\right)_{\omega},\left(\eta_{n}\right)_{\omega}\right\rangle_{\mathcal{H}} \omega=\lim _{n \rightarrow \omega}\left\langle\xi_{n}, \eta_{n}\right\rangle_{\mathcal{H}}$. The standard Hilbert space $\mathrm{L}^{2}\left(M^{\omega}\right)$ can be embedded into $\mathcal{H}^{\omega}$ as a closed subspace via the mapping $\mathrm{L}^{2}\left(M^{\omega}\right) \rightarrow \mathcal{H}^{\omega}:\left(x_{n}\right)^{\omega} \xi_{\varphi^{\omega}} \mapsto\left(x_{n} \xi_{\varphi}\right)_{\omega}$. For more on ultraproduct von Neumann algebras, we refer the reader to $\mathrm{AH12}$, Oc85.

In Section 4, we will need the following well-known fact about ultraproducts of semifinite von Neumann algebras. Let $(M, \operatorname{Tr})$ be any semifinite $\sigma$-finite von Neumann endowed with a faithful normal semifinite trace. Then the ultraproduct von Neumann algebra $M^{\omega}$ is semifinite and the weight $\operatorname{Tr} \circ \mathrm{E}_{\omega}$ is tracial on $M^{\omega}$ (see [AH12, Lemma 4.26]).

In Appendix A, we will need the following result about the centralizer $\left(M^{\omega}\right)^{\varphi^{\omega}}$ of the ultraproduct state $\varphi^{\omega}$.

Proposition 2.8. Let $(M, \varphi)$ be any $\sigma$-finite von Neumann algebra endowed with a faithful normal state and $\omega \in \beta(\mathbf{N}) \backslash \mathbf{N}$ any nonprincipal ultrafilter.

(1) If $M \neq \mathbf{C} 1$, then $\left(M^{\omega}\right)^{\varphi^{\omega}} \neq \mathbf{C} 1$.

(2) If $M$ is diffuse, then $\left(M^{\omega}\right)^{\varphi^{\omega}}$ is diffuse.

Proof. (1) Assume that $M \neq \mathbf{C} 1$. If $M^{\varphi} \neq \mathbf{C} 1$, then we also have $\left(M^{\omega}\right)^{\varphi^{\omega}} \neq \mathbf{C} 1$ since $M^{\varphi} \subset\left(M^{\omega}\right)^{\varphi^{\omega}}$. If $M^{\varphi}=\mathbf{C} 1$, then $M$ is a type $\mathrm{III}_{1}$ factor by [AH12, Lemma 5.3]. By [AH12, Theorem 4.20], $\left(M^{\omega}\right)^{\varphi^{\omega}}$ is a type $\mathrm{II}_{1}$ factor and hence $\left(M^{\omega}\right)^{\varphi^{\omega}} \neq \mathbf{C} 1$.

(2) Fix a sequence $\left(z_{n}\right)_{n}$ of central projections in $\mathcal{Z}(M)$ such that $\sum_{n} z_{n}=1, M z_{0}$ has a diffuse center and $M z_{n}$ is a diffuse factor for every $n \geq 1$. Observe that $\mathcal{Z}\left(M z_{0}\right) \subset M^{\varphi} z_{0}$ and hence $M^{\varphi} z_{0}$ is diffuse. Next, fix $n \geq 1$ such that $z_{n} \neq 0$ and put $\varphi_{z_{n}}=\frac{\varphi\left(z_{n} \cdot z_{n}\right)}{\varphi\left(z_{n}\right)} \in\left(M z_{n}\right)_{*}$. If $M z_{n}$ is a semifinite factor, then $M^{\varphi} z_{n}=\left(M z_{n}\right)^{\varphi_{z_{n}}}$ is diffuse. If $M z_{n}$ is a type $\mathrm{III}_{\lambda}$ factor, with $0 \leq \lambda<1$, then $M^{\varphi} z_{n}=\left(M z_{n}\right)^{\varphi_{z_{n}}}$ is diffuse by [Co72, Théorème 4.2.1 and Théorème 5.2.1]. If $M z_{n}$ is a type $\mathrm{III}_{1}$ factor, then $\left(M^{\omega}\right)^{\varphi^{\omega}} z_{n}=\left(\left(M z_{n}\right)^{\omega}\right)^{\varphi_{z_{n}}^{\omega}}$ is a type $\mathrm{II}_{1}$ factor by [AH12, 
Theorem 4.20]. We finally obtain that $\left(M^{\omega}\right)^{\varphi^{\omega}} z_{n}=\left(\left(M z_{n}\right)^{\omega}\right)^{\varphi_{z_{n}}^{\omega}}$ is diffuse for all $n$ and hence $\left(M^{\omega}\right)^{\varphi^{\omega}}$ is diffuse.

\section{Asymptotic ORThogonality PROPERTY}

The phenomenon of asymptotic orthogonality property inside free group factors was discovered by Popa in his seminal work [Po83, Lemma 2.1]. The main result of this section is the following optimal asymptotic orthogonality property result inside arbitrary free product von Neumann algebras. To fix notation, for each $i \in\{1,2\}$, let $\left(M_{i}, \varphi_{i}\right)$ be any $\sigma$-finite von Neumann algebra endowed with a faithful normal state. Denote by $(M, \varphi)=\left(M_{1}, \varphi_{1}\right) *\left(M_{2}, \varphi_{2}\right)$ the free product. As usual, denote by $\mathrm{E}_{M_{1}}: M \rightarrow M_{1}$ the unique $\varphi$-preserving conditional expectation. Let $Q \subset M_{1}$ be any diffuse von Neumann subalgebra with expectation. Fix a faithful state $\psi \in M_{*}$ such that $\sigma_{t}^{\psi}(Q)=Q$ and $\sigma_{t}^{\psi}\left(M_{1}\right)=M_{1}$ for all $t \in \mathbf{R}$. Observe that $\psi=\psi \circ \mathrm{E}_{M_{1}}$.

Theorem 3.1 below is a simultaneous generalization of [Ue10, Proposition 3.5] (which only deals with $y \in \operatorname{ker}\left(\varphi_{2}\right)$ ) and [Ho14, Theorem 3.1] (which requires the centralizer $\left(M_{1}\right)^{\varphi_{1}}$ to be diffuse).

Theorem 3.1. Keep the same notation as above. For all $x \in Q^{\prime} \cap M^{\omega}$ and all $y, z \in M \ominus M_{1}$, the vectors

$$
y\left(x-\mathrm{E}_{M_{1}^{\omega}}(x)\right) \xi_{\psi^{\omega}},\left(y \mathrm{E}_{M_{1}^{\omega}}(x)-\mathrm{E}_{M_{1}^{\omega}}(x) z\right) \xi_{\psi^{\omega}} \text { and }\left(\mathrm{E}_{M_{1}^{\omega}}(x)-x\right) z \xi_{\psi^{\omega}}
$$

are mutually orthogonal in the standard Hilbert space $\mathrm{L}^{2}\left(M^{\omega}\right)$ where $\xi_{\psi^{\omega}} \in \mathfrak{P}^{M^{\omega}}$ is the canonical representing vector of the ultraproduct state $\psi^{\omega}$.

Proof. The proof of Theorem 3.1 is a reconstruction of [Ho14, Theorem 3.1] and the new input is the 'state replacement' procedure developed in [Ue10].

Let $\left(M^{\omega}, \mathrm{L}^{2}\left(M^{\omega}\right), J^{M^{\omega}}, \mathfrak{P}^{M^{\omega}}\right)$ be the standard form of the ultraproduct von Neumann algebra $M^{\omega}$, which is known to be obtained from the standard form $\left(M, \mathrm{~L}^{2}(M), J^{M}, \mathfrak{P}^{M}\right)$ of the original von Neumann algebra $M$ in a rather canonical fashion (see [AH12, Corollary 3.27]). It suffices to prove, instead of the original assertion, that, for all $z^{\prime} \in M \ominus M_{1}$ with the given $x, y$ in the original assertion, the vectors

$$
y\left(x-\mathrm{E}_{M_{1}^{\omega}}(x)\right) \xi_{\psi^{\omega}},\left(y \mathrm{E}_{M_{1}^{\omega}}(x)-J^{M^{\omega}} z^{\prime} J^{M^{\omega}} \mathrm{E}_{M_{1}^{\omega}}(x)\right) \xi_{\psi^{\omega}} \text { and } J^{M^{\omega}} z^{\prime} J^{M^{\omega}}\left(\mathrm{E}_{M_{1}^{\omega}}(x)-x\right) \xi_{\psi^{\omega}}
$$

are mutually orthogonal in the standard Hilbert space $\mathrm{L}^{2}\left(M^{\omega}\right)$. In fact, by a standard approximation argument we may and do assume that the given $z$ in the original assertion is analytic with respect to the modular automorphism group $\sigma^{\psi}$. By [AH12, Theorem 4.1] together with [Ta03, Lemma VIII.3.18 (ii)], we have

$$
\begin{aligned}
\mathrm{E}_{M_{1}^{\omega}}(x) z \xi_{\psi^{\omega}} & =J^{M^{\omega}} \sigma_{\mathrm{i} / 2}^{\psi}(z)^{*} J^{M^{\omega}} \mathrm{E}_{M_{1}^{\omega}}(x) \xi_{\psi^{\omega}}, \\
\left(\mathrm{E}_{M_{1}^{\omega}}(x)-x\right) z \xi_{\psi^{\omega}} & =J^{M^{\omega}} \sigma_{\mathrm{i} / 2}^{\psi}(z)^{*} J^{M^{\omega}}\left(\mathrm{E}_{M_{1}^{\omega}}(x)-x\right) \xi_{\psi^{\omega}},
\end{aligned}
$$

so that the above new assertion immediately gives the desired one.

For all $i \in\{1,2\}$, denote by $A_{i} \subset M_{i}$ the $\sigma$-weakly dense unital $*$-subalgebra of all the analytic elements in $M_{i}$ with respect to the modular automorphism group $\sigma^{\varphi_{i}}$ and write $A_{i}^{\circ}:=A_{i} \cap M_{i}^{\circ}$ with the standard notation $M_{i}^{\circ}:=\operatorname{ker}\left(\varphi_{i}\right)$. As in the proof of [Ho14, Theorem 3.1], we may and will assume that the elements $y$ and $z^{\prime}$ are analytic with respect to the modular automorphism group $\sigma^{\varphi}$ and $y$ and $\sigma_{\mathrm{i} / 2}^{\varphi}\left(z^{\prime}\right)^{*}$ are finite sums of reduced words $w_{1}, \ldots, w_{\ell}$ and $w_{1}^{\prime}, \ldots, w_{\ell^{\prime}}^{\prime}$ in $A_{1} A_{2}^{\circ} \cdots A_{2}^{\circ} A_{1}$, respectively. Unlike usual, we call an element in $M_{1} M_{2}^{\circ} \cdots M_{2}^{\circ} M_{1}$ a reduced word in what follows.

Let $V$ be the finite dimensional subspace of $M_{1}$ obtained by looking at the letters coming from $A_{1}^{\circ} \cup\{1\}$ appearing in $y$ in the same fashion as in the proof of [Ho14, Theorem 3.1]. Namely, $V$ is the linear span of the following $A_{1}$-letters: 
- the leftmost $A_{1}$-letters of the reduced words $w_{i}, w_{i}^{*}, 1 \leq i \leq \ell$;

- the rightmost $A_{1}$-letters of the reduced words $w_{i}^{\prime}, \sigma_{-\mathrm{i}}^{\varphi}\left(w_{i}^{\prime *}\right), 1 \leq i \leq \ell^{\prime}$;

- the leftmost $A_{1}$-letters of all the reduced words appearing in the elements $w_{i}^{*} w_{j}, 1 \leq$ $i, j \leq \ell$;

- the rightmost $A_{1}$-letters of all the reduced words appearing in the elements $w_{i}^{\prime} \sigma_{-\mathrm{i}}^{\varphi}\left(w_{j}^{\prime *}\right)$, $1 \leq i, j \leq \ell^{\prime}$.

Choose an orthonormal basis $e_{1}, \ldots, e_{m}$ of $V$ with respect to the inner product $(a \mid b)_{\varphi_{1}}:=$ $\varphi_{1}\left(b^{*} a\right)$ on $M_{1}$. Denote by $W$ the range of the mapping $a \in M_{1} \mapsto a-\sum_{i=1}^{m}\left(a \mid e_{i}\right)_{\varphi_{1}} e_{i} \in M_{1}$. It follows that $M_{1}=V+W$ is an orthogonal decomposition with respect to the inner product $(\cdot \mid \cdot)_{\varphi_{1}}$ defined on $M_{1}$ as above.

Let $\mathfrak{H}$ be the direct sum of all the alternating tensor products in $\mathrm{L}^{2}\left(M_{1}\right)^{\circ}$ and $\mathrm{L}^{2}\left(M_{2}\right)^{\circ}$ starting and ending with $\mathrm{L}^{2}\left(M_{2}\right)^{\circ}$. Here $\mathrm{L}^{2}\left(M_{i}\right)^{\circ}$ denotes the orthogonal complement of the canonical representing vector $\xi_{\varphi_{i}} \in \mathfrak{P}^{M_{i}}$ of the given state $\varphi_{i}$. Thanks to $*-\operatorname{alg}\left(M_{1}, M_{2}\right)=$ $M_{1}+\operatorname{span}\left(M_{1} M_{2}^{\circ} \cdots M_{2}^{\circ} M_{1}\right)$ together with the formula of modular conjugation (see Ue98a, Proposition II-C]), the standard Hilbert space $\mathrm{L}^{2}(M)$ is naturally identified with $\mathrm{L}^{2}\left(M_{1}\right) \oplus$ $\mathrm{L}^{2}\left(M_{1}\right) \otimes \mathfrak{H} \otimes \mathrm{L}^{2}\left(M_{1}\right)$ as $M_{1}-M_{1}$-bimodules. Decompose $\mathrm{L}^{2}\left(M_{1}\right) \otimes \mathfrak{H} \otimes \mathrm{L}^{2}\left(M_{1}\right)$ into three subspaces $\mathcal{K}_{1}, \mathcal{K}_{2}, \mathcal{L}$ defined by

$$
\begin{aligned}
\mathcal{K}_{1} & :=\left(V \xi_{\varphi_{1}}\right) \otimes \mathfrak{H} \otimes \mathrm{L}^{2}\left(M_{1}\right), \\
\mathcal{K}_{2} & :=\left(\overline{W \xi_{\varphi_{1}}}\right) \otimes \mathfrak{H} \otimes\left(V \xi_{\varphi_{1}}\right), \\
\mathcal{L} & :=\left(\overline{W \xi_{\varphi_{1}}}\right) \otimes \mathfrak{H} \otimes\left(\overline{W \xi_{\varphi_{1}}}\right) .
\end{aligned}
$$

It is clear that these subspaces are generated by

$$
\begin{aligned}
& V M_{2}^{\circ} \cdots M_{2}^{\circ} M_{1} \xi_{\varphi}, \\
& W M_{2}^{\circ} \cdots M_{2}^{\circ} V \xi_{\varphi}, \\
& W M_{2}^{\circ} \cdots M_{2}^{\circ} W \xi_{\varphi},
\end{aligned}
$$

respectively, in $\mathrm{L}^{2}(M)$, where $\xi_{\varphi} \in \mathfrak{P}^{M}$ is the canonical representing vector of the free product state $\varphi$. Remark that the direct summand $\mathrm{L}^{2}\left(M_{1}\right)$ in $\mathrm{L}^{2}(M)$ is given by $\overline{M_{1} \xi_{\varphi}}=\overline{M_{1} \xi_{\psi}}$ thanks to $\psi \circ \mathrm{E}_{M_{1}}=\psi$ (see e.g. [Ko88, Appendix I]).

Let $\delta>0$ be arbitrarily chosen. By Lemma 2.1, choose a faithful state $\phi_{1} \in Q_{*}$ such that $\left\|\left.\psi\right|_{Q}-\phi_{1}\right\|<\delta$ and $Q^{\phi_{1}}$ is diffuse. Denote by $\mathrm{E}_{Q}^{M_{1}}: M_{1} \rightarrow Q$ the unique $\psi$-preserving conditional expectation and put $\phi:=\phi_{1} \circ \mathrm{E}_{Q}^{M_{1}} \circ \mathrm{E}_{M_{1}}$. Then we have $\phi=\phi \circ \mathrm{E}_{M_{1}}, Q^{\phi}$ is diffuse and $\|\psi-\phi\|=\left\|\left.\psi\right|_{Q}-\phi_{1}\right\|<\delta$ so that the canonical representing vectors $\xi_{\psi}, \xi_{\phi} \in \mathfrak{P}^{M}$ of the states $\psi, \phi$ satisfy $\left\|\xi_{\psi}-\xi_{\phi}\right\|_{\mathrm{L}^{2}(M)}<\delta^{1 / 2}$ by the Araki-Powers-Størmer inequality (see [Ta03, Theorem IX.1.2 (iv)]). In what follows, we denote by $P_{\mathcal{X}}$ the orthogonal projection from $\mathrm{L}^{2}(M)$ onto a (closed) subspace $\mathcal{X}$.

Let $\left(x_{n}\right)_{n} \in \mathcal{M}^{\omega}(M)$ such that $x=\left(x_{n}\right)^{\omega}$ with $C:=\sup _{n}\left\|x_{n}\right\|_{\infty}$. Then for all $n \in \mathbf{N}$ and all $i \in\{1,2\}$, we have

$$
\left\|P_{\mathcal{K}_{i}} x_{n} \xi_{\psi}\right\|_{\mathrm{L}^{2}(M)}<C \delta^{1 / 2}+\left\|P_{\mathcal{K}_{i}} x_{n} \xi_{\phi}\right\|_{\mathrm{L}^{2}(M)} .
$$

For a while, we will be working with $\left\|P_{\mathcal{K}_{i}} x_{n} \xi_{\phi}\right\|_{\mathrm{L}^{2}(M)}$ by the same method used in the proof of [Ho14, Theorem 3.1]. Since $Q^{\phi}$ is diffuse, we can choose a unitary $u \in \mathcal{U}\left(Q^{\phi}\right)$ such that $\lim _{k \rightarrow \pm \infty} u^{k}=0 \sigma$-weakly. Consider the unitary transformation $T: \mathrm{L}^{2}(M) \rightarrow \mathrm{L}^{2}(M): \xi \rightarrow$ $u J^{M} u J^{M} \xi=: u \cdot \xi \cdot u^{*}$. Observe that since $u \in \mathcal{U}\left(M^{\phi}\right)$ and hence $\left[u, \xi_{\phi}\right]=0$, for all $n \in \mathbf{N}$, all $i \in\{1,2\}$ and all $k \in \mathbf{Z}$, we have

$$
T^{k} P_{\mathcal{K}_{i}} x_{n} \xi_{\phi}=u^{k} \cdot\left(P_{\mathcal{K}_{i}} x_{n} \xi_{\phi}\right) \cdot u^{-k}=P_{u^{k} \cdot \mathcal{K}_{i} \cdot u^{-k}} u^{k} x_{n} u^{-k} \xi_{\phi}=P_{T^{k} \mathcal{K}_{i}} u^{k} x_{n} u^{-k} \xi_{\phi} .
$$

Here is a simple claim, which is just a reconstruction of Claim 1 of [Ho14, §3]. 
Claim. For any $\varepsilon>0$, there exists $k_{0} \in \mathbb{N}$ such that for all $i \in\{1,2\}$, all $\xi, \eta \in \mathcal{K}_{i}$ and all $k \geq k_{0}$, we have $\left|\left\langle T^{k} \xi, \eta\right\rangle_{\mathrm{L}^{2}(M)}\right| \leq \varepsilon\|\xi\|_{\mathrm{L}^{2}(M)}\|\eta\|_{\mathrm{L}^{2}(M)}$, that is, $T^{k} \mathcal{K}_{i} \perp_{\varepsilon} \mathcal{K}_{i}$ in the sense of Ho12a, Definition 2.1].

Proof of the Claim. Denote by $J^{M_{1}}$ the modular conjugation on $\mathrm{L}^{2}\left(M_{1}\right)$. For $\xi=\sum_{i=1}^{m}\left(e_{i} \xi_{\varphi_{1}}\right) \otimes$ $\xi_{i}, \eta=\sum_{j=1}^{m}\left(e_{j} \xi_{\varphi_{1}}\right) \otimes \eta_{j} \in \mathcal{K}_{1}$ inside $\mathrm{L}^{2}\left(M_{1}\right) \otimes\left(\mathfrak{H} \otimes \mathrm{L}^{2}\left(M_{1}\right)\right)$, we have

$$
\begin{aligned}
\left|\left\langle T^{k} \xi, \eta\right\rangle_{\mathrm{L}^{2}(M)}\right| & \leq \sum_{i, j=1}^{m}\left|\left(u^{k} e_{i} \mid e_{j}\right)_{\varphi_{1}}\right|\left\|\xi_{i}\right\|_{\mathrm{L}^{2}(M)}\left\|\eta_{j}\right\|_{\mathrm{L}^{2}(M)} \\
& \leq \max _{1 \leq i, j \leq m}\left|\left(u^{k} e_{i} \mid e_{j}\right)_{\varphi_{1}}\right| \times\|\xi\|_{\mathrm{L}^{2}(M)}\|\eta\|_{\mathrm{L}^{2}(M)} .
\end{aligned}
$$

Similarly, for $\xi^{\prime}=\sum_{i=1}^{m} \xi_{i}^{\prime} \otimes\left(e_{i} \xi_{\varphi_{1}}\right), \eta^{\prime}=\sum_{j=1}^{m} \eta_{j}^{\prime} \otimes\left(e_{j} \xi_{\varphi_{1}}\right) \in \mathcal{K}_{2}$ inside $\left(\mathrm{L}^{2}\left(M_{1}\right) \otimes \mathfrak{H}\right) \otimes \mathrm{L}^{2}\left(M_{1}\right)$, we have

$$
\begin{aligned}
\left|\left\langle T^{k} \xi^{\prime}, \eta^{\prime}\right\rangle_{\mathrm{L}^{2}(M)}\right| & \leq \sum_{i, j=1}^{m}\left\|\xi_{i}^{\prime}\right\|_{\mathrm{L}^{2}(M)}\left\|\eta_{j}^{\prime}\right\|_{\mathrm{L}^{2}(M)}\left|\left\langle u^{-k} J^{M_{1}} e_{j} \xi_{\varphi_{1}}, J^{M_{1}} e_{i} \xi_{\varphi_{1}}\right\rangle_{\mathrm{L}^{2}\left(M_{1}\right)}\right| \\
& \leq \max _{1 \leq i, j \leq m}\left|\left\langle u^{-k} J^{M_{1}} e_{j} \xi_{\varphi_{1}}, J^{M_{1}} e_{i} \xi_{\varphi_{1}}\right\rangle_{\mathrm{L}^{2}\left(M_{1}\right)}\right| \times\left\|\xi^{\prime}\right\|_{\mathrm{L}^{2}(M)}\left\|\eta^{\prime}\right\|_{\mathrm{L}^{2}(M)}
\end{aligned}
$$

These two facts together with $\lim _{k \rightarrow \pm \infty} u^{k}=0 \sigma$-weakly imply the desired assertion.

Combining Equation (3.2) with the parallelogram law, for all $n \in \mathbf{N}$, all $i \in\{1,2\}$ and all $k \in \mathbf{Z}$, we have

$$
\begin{aligned}
\left\|P_{\mathcal{K}_{i}} x_{n} \xi_{\phi}\right\|_{\mathrm{L}^{2}(M)}^{2} & =\left\|T^{k} P_{\mathcal{K}_{i}} x_{n} \xi_{\phi}\right\|_{\mathrm{L}^{2}(M)}^{2} \\
& \leq 2\left\|\left(u^{k} x_{n} u^{-k}-x_{n}\right) \xi_{\phi}\right\|_{\mathrm{L}^{2}(M)}^{2}+2\left\|P_{T^{k} \mathcal{K}_{i}} x_{n} \xi_{\phi}\right\|_{\mathrm{L}^{2}(M)}^{2} .
\end{aligned}
$$

Thanks to this and the above Claim and since $x \in Q^{\prime} \cap M^{\omega}$, the $\varepsilon$-orthogonality technique from [Ho12a, Proposition 2.3] works to show that $\lim _{n \rightarrow \omega}\left\|P_{\mathcal{K}_{i}} x_{n} \xi_{\phi}\right\|_{\mathrm{L}^{2}(M)}=0$ in the same way as in the proof of Claim 2 in [Ho14, §3]. Consequently, we have $\lim _{n \rightarrow \omega}\left\|P_{\mathcal{K}_{i}} x_{n} \xi_{\psi}\right\|_{L^{2}(M)} \leq C \delta^{1 / 2}$. Since $\delta>0$ can be arbitrarily small, we finally obtain

$$
\lim _{n \rightarrow \omega}\left\|P_{\mathcal{K}_{i}} x_{n} \xi_{\psi}\right\|_{L^{2}(M)}=0, \forall i \in\{1,2\} \text {. }
$$

It is standard, see $\mathrm{AH} 12$, Theorem 3.7], that $\mathrm{L}^{2}\left(M^{\omega}\right)$ is embedded into the ultraproduct Hilbert space $\mathrm{L}^{2}(M)^{\omega}$ by $\left(a_{n}\right)^{\omega} \xi_{\varphi^{\omega}} \mapsto\left(a_{n} \xi_{\varphi}\right)_{\omega}$ for $\left(a_{n}\right)^{\omega} \in M^{\omega}$ with representing sequence $\left(a_{n}\right)_{n} \in \mathcal{M}^{\omega}(M)$. Remark that the other mapping $\left(a_{n}\right)^{\omega} \xi_{\psi^{\omega}} \mapsto\left(a_{n} \xi_{\psi}\right)_{\omega}$ gives exactly the same embedding since we already fix the choice (or realization) of standard forms. By (3.3) together with [AH12, Proposition 3.15, Corollary 3.27, Corollary 3.28], we obtain

$$
\begin{aligned}
y\left(x-\mathrm{E}_{M_{1}^{\omega}}(x)\right) \xi_{\psi^{\omega}} & =\left(y P_{\mathcal{L}} x_{n} \xi_{\psi}\right)_{\omega}, \\
\left(y \mathrm{E}_{M_{1}^{\omega}}(x)-J^{M^{\omega}} z^{\prime} J^{M^{\omega}} \mathrm{E}_{M_{1}^{\omega}}(x)\right) \xi_{\psi^{\omega}} & =\left(\left(y \mathrm{E}_{M_{1}}\left(x_{n}\right)-J^{M} z^{\prime} J^{M} \mathrm{E}_{M_{1}}\left(x_{n}\right)\right) \xi_{\psi}\right)_{\omega}, \\
J^{M^{\omega}} z^{\prime} J^{M^{\omega}}\left(\mathrm{E}_{M_{1}^{\omega}}(x)-x\right) \xi_{\psi^{\omega}} & =\left(-J^{M} z^{\prime} J^{M} P_{\mathcal{L}} x_{n} \xi_{\psi}\right)_{\omega}
\end{aligned}
$$

inside $\mathrm{L}^{2}(M)^{\omega}$. Note that $y P_{\mathcal{L}} x_{n} \xi_{\psi}$ sits in the closed linear span of $w_{i} W M_{2}^{\circ} \cdots M_{2}^{\circ} W \xi_{\varphi}, 1 \leq$ $i \leq \ell$, and $J^{M} z^{\prime} J^{M} P_{\mathcal{L}} x_{n} \xi_{\psi}$ sits in the closed linear span of $W M_{2}^{\circ} \cdots M_{2}^{\circ} W w_{j}^{\prime} \xi_{\varphi}, 1 \leq j \leq$ $\ell^{\prime}$. Moreover, note that $\left(y \mathrm{E}_{M_{1}}\left(x_{n}\right)-J^{M} z^{\prime} J^{M} \mathrm{E}_{M_{1}}\left(x_{n}\right)\right) \xi_{\psi} \in\left(y+J^{M} z^{\prime} J^{M}\right) \overline{M_{1} \xi_{\psi}}=(y+$ $\left.J^{M} z^{\prime} J^{M}\right) \overline{M_{1} \xi_{\varphi}}\left(n . b . \psi=\psi \circ \mathrm{E}_{M_{1}}\right)$ as well as that $J^{M} z^{\prime} J^{M} b \xi_{\varphi}=b \sigma_{\mathrm{i} / 2}^{\varphi}\left(z^{\prime}\right)^{*} \xi_{\varphi}$ for every $b \in M_{1}$ by Ta03, Lemma VIII.3.18 (ii)]. This shows that $\left(y \mathrm{E}_{M_{1}}\left(x_{n}\right)-J^{M} z^{\prime} J^{M} \mathrm{E}_{M_{1}}\left(x_{n}\right)\right) \xi_{\psi}$ sits in the closed linear span of $\left(w_{i} M_{1}+M_{1} w_{j}^{\prime}\right) \xi_{\varphi}, 1 \leq i \leq \ell$ and $1 \leq j \leq \ell^{\prime}$.

Observe that the choice of $V$ makes the subspaces $w_{i} W M_{2}^{\circ} \cdots M_{2}^{\circ} W \xi_{\varphi}, W M_{2}^{\circ} \cdots M_{2}^{\circ} W w_{j}^{\prime} \xi_{\varphi}$, $\left(w_{i} M_{1}+M_{1} w_{j}^{\prime}\right) \xi_{\varphi}$ mutually orthogonal for all $1 \leq i \leq \ell$ and all $1 \leq j \leq \ell^{\prime}$. This can 
easily be checked exactly in the same way as in Claim 3 of [Ho14, §3] (which looks complicated but not difficult). Therefore, $y\left(x-\mathrm{E}_{M_{1}^{\omega}}(x)\right) \xi_{\psi^{\omega}},\left(y \mathrm{E}_{M_{1}^{\omega}}(x)-J^{M^{\omega}} z^{\prime} J^{M^{\omega}} \mathrm{E}_{M_{1}^{\omega}}(x)\right) \xi_{\psi^{\omega}}$ and $J^{M^{\omega}} z^{\prime} J^{M^{\omega}}\left(\mathrm{E}_{M_{1}^{\omega}}(x)-x\right) \xi_{\psi^{\omega}}$ are mutually orthogonal in $\mathrm{L}^{2}\left(M^{\omega}\right)$. This finishes the proof of Theorem 3.1

\section{Proofs of Theorem A and Corollary B}

A key deformation/rigidity result for semifinite von Neumann algebras. Theorem 4.1 below relies on Popa's deformation/rigidity theory [Po01, $\mathrm{Po} 03$, Po06] and is an adaptation of Peterson's L L ${ }^{2}$-rigidity results [Pe06, Theorems 4.3 and 4.5] for semifinite von Neumann algebras using Popa's malleable deformations instead of Peterson's $\mathrm{L}^{2}$-derivations.

Recall from Po03, Po06 that for any inclusion $\mathcal{M} \subset \widetilde{\mathcal{M}}$ of semifinite von Neumann algebras with trace preserving conditional expectation, a trace preserving action $\mathbf{R} \rightarrow \operatorname{Aut}(\widetilde{\mathcal{M}}): t \mapsto \theta_{t}$ is called a malleable deformation if there exists a period two trace preserving $*$-automorphism $\beta \in \operatorname{Aut}(\widetilde{\mathcal{M}})$ such that $\beta \circ \theta_{t}=\theta_{-t} \circ \beta$ for all $t \in \mathbf{R}$. Denote by $\mathrm{E}_{\mathcal{M}}: \widetilde{\mathcal{M}} \rightarrow \mathcal{M}$ the unique trace preserving conditional expectation. We will simply denote by $\|\cdot\|_{2}$ the $\mathrm{L}^{2}$-norm associated with the ambient faithful normal semifinite trace. By [Po06, Lemma 2.1], any malleable deformation automatically satisfies the following transversality property:

$$
\left\|x-\theta_{2 t}(x)\right\|_{2} \leq 2\left\|\theta_{t}(x)-\mathrm{E}_{\mathcal{M}}\left(\theta_{t}(x)\right)\right\|_{2}, \forall x \in \mathcal{M} \cap \mathrm{L}^{2}(\mathcal{M}, \operatorname{Tr}) .
$$

The main result of this subsection is the following uniform convergence theorem for malleable deformations.

Theorem 4.1. Let $\mathcal{B} \subset \mathcal{M} \subset \widetilde{\mathcal{M}}$ be an inclusion of semifinite von Neumann algebras with trace preserving conditional expectations. Let $\mathbf{R} \rightarrow \operatorname{Aut}(\widetilde{\mathcal{M}}): t \mapsto \theta_{t}$ be a trace preserving malleable deformation. Let $p \in \mathcal{M}$ be any nonzero finite trace projection and $\mathcal{Q} \subset p \mathcal{M} p$ any von Neumann subalgebra. Assume that the following conditions hold:

(i) The $p \mathcal{M} p$-p $\mathcal{M} p$-bimodule $\mathrm{L}^{2}(p \widetilde{\mathcal{M}} p) \ominus \mathrm{L}^{2}(p \mathcal{M} p)$ is weakly contained in the coarse $p \mathcal{M} p$ $p \mathcal{M} p$-bimodule $\mathrm{L}^{2}(p \mathcal{M} p) \otimes \mathrm{L}^{2}(p \mathcal{M} p)$.

(ii) The von Neumann algebra $\mathcal{Q}$ has no amenable direct summand.

(iii) There exists a nonprincipal ultrafilter $\omega \in \beta(\mathbf{N}) \backslash \mathbf{N}$ such that $\mathcal{Q}^{\prime} \cap(p \mathcal{M} p)^{\omega} \npreceq \mathcal{M}^{\omega} \mathcal{B}^{\omega}$.

(iv) Denote by $\mathrm{E}_{\mathcal{B}}: \mathcal{M} \rightarrow \mathcal{B}$ the unique trace preserving conditional expectation. For every net $\left(v_{i}\right)_{i \in I}$ of unitaries in $\mathcal{U}(p \mathcal{M} p)$ satisfying $\lim _{i}\left\|\mathrm{E}_{\mathcal{B}}\left(b^{*} v_{i} a\right)\right\|_{2}=0$ for all $a, b \in p \mathcal{M}$, we have $\lim _{i}\left\|\mathrm{E}_{\mathcal{M}}\left(d^{*} v_{i} c\right)\right\|_{2}=0$ for all $c, d \in p(\widetilde{\mathcal{M}} \ominus \mathcal{M})$.

Then the map $\mathbf{R} \rightarrow \operatorname{Aut}(\widetilde{\mathcal{M}}): t \mapsto \theta_{t}$ converges uniformly on $\operatorname{Ball}(\mathcal{Q})$ in $\|\cdot\|_{2}$ as $t \rightarrow 0$.

Proof. Put $\mathcal{P}=\mathcal{Q}^{\prime} \cap(p \mathcal{M} p)^{\omega}$. For every $t \in \mathbf{R}$, define $\theta_{t}^{\omega} \in \operatorname{Aut}\left(\widetilde{\mathcal{M}}^{\omega}\right)$ by $\theta_{t}^{\omega}\left(\left(x_{n}\right)^{\omega}\right)=\left(\theta_{t}\left(x_{n}\right)\right)^{\omega}$. We note that the map $\mathbf{R} \rightarrow \operatorname{Aut}\left(\widetilde{\mathcal{M}}^{\omega}\right): t \mapsto \theta_{t}^{\omega}$ need not be continuous. However, exploiting Popa's spectral gap argument [Po06], we can show the following uniform convergence result.

Claim. The map $\mathbf{R} \rightarrow \operatorname{Aut}\left(\widetilde{\mathcal{M}}^{\omega}\right): t \mapsto \theta_{t}^{\omega}$ converges uniformly on $\operatorname{Ball}(\mathcal{P})$ in $\|\cdot\|_{2}$ as $t \rightarrow 0$.

Proof of Claim. For the Claim, we will only use Conditions (i),(ii). Assume by contradiction that the map $\mathbf{R} \rightarrow \operatorname{Aut}\left(\widetilde{\mathcal{M}}^{\omega}\right): t \mapsto \theta_{t}^{\omega}$ does not converge uniformly on $\operatorname{Ball}(\mathcal{P})$ in $\|\cdot\|_{2}$ as $t \rightarrow 0$. Thus there exist $c>0$, a sequence $\left(t_{k}\right)_{k}$ of positive reals such that $\lim _{k} t_{k}=0$ and a sequence $\left(X_{k}\right)_{k}$ in $\operatorname{Ball}(\mathcal{P})$ such that $\left\|X_{k}-\theta_{2 t_{k}}^{\omega}\left(X_{k}\right)\right\|_{2} \geq 2 c$ for all $k \in \mathbf{N}$. Write $X_{k}=\left(x_{n}^{(k)}\right)^{\omega}$ with $x_{n}^{(k)} \in \operatorname{Ball}(p \mathcal{M} p)$ satisfying $\lim _{n \rightarrow \omega}\left\|y x_{n}^{(k)}-x_{n}^{(k)} y\right\|_{2}=0$ and $2 c \leq\left\|X_{k}-\theta_{2 t_{k}}^{\omega}\left(X_{k}\right)\right\|_{2}=$ $\lim _{n \rightarrow \omega}\left\|x_{n}^{(k)}-\theta_{2 t_{k}}\left(x_{n}^{(k)}\right)\right\|_{2}$ for all $k \in \mathbf{N}$ and all $y \in \mathcal{Q}$. 
Denote by $I$ the directed set of all pairs $(\mathcal{F}, \varepsilon)$ with $\mathcal{F} \subset \operatorname{Ball}(\mathcal{Q})$ finite subset and $\varepsilon>0$. Let $i=(\mathcal{F}, \varepsilon) \in I$ and put $\delta=\min \left(\frac{\varepsilon}{6}, \frac{c}{8}\right)$. Choose $k \in \mathbf{N}$ large enough so that $\left\|p-\theta_{t_{k}}(p)\right\|_{2} \leq \delta$ and $\left\|a-\theta_{t_{k}}(a)\right\|_{2} \leq \varepsilon / 6$ for all $a \in \mathcal{F}$. Then choose $n \in \mathbf{N}$ large enough so that $\left\|x_{n}^{(k)}-\theta_{2 t_{k}}\left(x_{n}^{(k)}\right)\right\|_{2} \geq$ $c$ and $\left\|a x_{n}^{(k)}-x_{n}^{(k)} a\right\|_{2} \leq \varepsilon / 3$ for all $a \in \mathcal{F}$.

Put $\xi_{i}=\theta_{t_{k}}\left(x_{n}^{(k)}\right)-\mathrm{E}_{\mathcal{M}}\left(\theta_{t_{k}}\left(x_{n}^{(k)}\right)\right) \in \mathrm{L}^{2}(\widetilde{\mathcal{M}}) \ominus \mathrm{L}^{2}(\mathcal{M})$ and $\eta_{i}=p \xi_{i} p \in \mathrm{L}^{2}(p \widetilde{\mathcal{M}} p) \ominus \mathrm{L}^{2}(p \mathcal{M} p)$. By the transversality property of the malleable deformation $\left(\theta_{t}\right)$, we have

$$
\left\|\xi_{i}\right\|_{2} \geq \frac{1}{2}\left\|x_{n}^{(k)}-\theta_{2 t_{k}}\left(x_{n}^{(k)}\right)\right\|_{2} \geq \frac{c}{2} .
$$

Observe that $\left\|p \theta_{t_{k}}\left(x_{n}^{(k)}\right) p-\theta_{t_{k}}\left(x_{n}^{(k)}\right)\right\|_{2} \leq 2\left\|p-\theta_{t_{k}}(p)\right\|_{2} \leq 2 \delta$. Since $p \in \mathcal{M}$, by Pythagoras theorem, we moreover have

$$
\left\|p \theta_{t_{k}}\left(x_{n}^{(k)}\right) p-\theta_{t_{k}}\left(x_{n}^{(k)}\right)\right\|_{2}^{2}=\left\|\mathrm{E}_{\mathcal{M}}\left(p \theta_{t_{k}}\left(x_{n}^{(k)}\right) p-\theta_{t_{k}}\left(x_{n}^{(k)}\right)\right)\right\|_{2}^{2}+\left\|\eta_{i}-\xi_{i}\right\|_{2}^{2}
$$

and hence $\left\|\eta_{i}-\xi_{i}\right\|_{2} \leq 2 \delta$. This implies that

$$
\left\|\eta_{i}\right\|_{2} \geq\left\|\xi_{i}\right\|_{2}-\left\|\eta_{i}-\xi_{i}\right\|_{2} \geq \frac{c}{2}-2 \delta \geq \frac{c}{4}
$$

For all $x \in p \mathcal{M} p$, we have

$$
\left\|x \eta_{i}\right\|_{2}=\left\|\left(1-\mathrm{E}_{\mathcal{M}}\right)\left(x \theta_{t_{k}}\left(x_{n}^{(k)}\right) p\right)\right\|_{2} \leq\left\|x \theta_{t_{k}}\left(x_{n}^{(k)}\right) p\right\|_{2} \leq\|x\|_{2} .
$$

By Popa's spectral gap argument [Po06], for all $a \in \mathcal{F} \subset \operatorname{Ball}(p \mathcal{M} p)$, we have

$$
\begin{aligned}
\left\|a \eta_{i}-\eta_{i} a\right\|_{2} & =\left\|\left(1-\mathrm{E}_{\mathcal{M}}\right)\left(a \theta_{t_{k}}\left(x_{n}^{(k)}\right) p-p \theta_{t_{k}}\left(x_{n}^{(k)}\right) a\right)\right\|_{2} \\
& \leq\left\|a \theta_{t_{k}}\left(x_{n}^{(k)}\right) p-p \theta_{t_{k}}\left(x_{n}^{(k)}\right) a\right\|_{2} \\
& \leq 2\left\|a-\theta_{t_{k}}(a)\right\|_{2}+2\left\|p-\theta_{t_{k}}(p)\right\|_{2}+\left\|a x_{n}^{(k)}-x_{n}^{(k)} a\right\|_{2} \\
& \leq \frac{\varepsilon}{3}+\frac{\varepsilon}{3}+\frac{\varepsilon}{3}=\varepsilon .
\end{aligned}
$$

Hence $\eta_{i} \in \mathrm{L}^{2}(p \widetilde{\mathcal{M}} p) \ominus \mathrm{L}^{2}(p \mathcal{M} p)$ is a net of vectors satisfying lim $\sup _{i}\left\|x \eta_{i}\right\|_{2} \leq\|x\|_{2}$ for all $x \in p \mathcal{M} p, \liminf _{i}\left\|\eta_{i}\right\|_{2} \geq \frac{c}{4}$ and $\lim _{i}\left\|a \eta_{i}-\eta_{i} a\right\|_{2}=0$ for all $a \in \mathcal{Q}$. By Condition (i), it follows that $\mathcal{Q} \subset p \mathcal{M} p$ has an amenable direct summand by Connes's characterization of amenability C075] for finite von Neumann algebras (see also [Io12, Lemma 2.3]). This is a contradiction to Condition (ii) and finishes the proof of the Claim.

Next, we use an idea due to Peterson Pe06 in combination with the above Claim to bring down the uniform convergence to $\operatorname{Ball}(\mathcal{Q})$. In what follows, we will use Conditions (iii),(iv). Let $\varepsilon>0$. By the above Claim, there exists $t_{0}>0$ such that $\left\|v-\theta_{t}^{\omega}(v)\right\|_{2}<\frac{\varepsilon^{2}}{16}$ for all $v \in \mathcal{U}(\mathcal{P})$ (recall $\mathcal{P}=\mathcal{Q}^{\prime} \cap(p \mathcal{M} p)^{\omega}$ ) and all $t \in\left[-t_{0}, t_{0}\right]$. Fix $x \in \operatorname{Ball}(\mathcal{Q})$ and $t \in\left[-t_{0}, t_{0}\right]$. We will show that $\left\|x-\theta_{2 t}(x)\right\|_{2} \leq \varepsilon$.

Denote by $I$ the directed set of all pairs $(\mathcal{F}, \delta)$ with $\mathcal{F} \subset \operatorname{Ball}(p \mathcal{M})$ finite subset and $\delta>0$. Fix $i=(\mathcal{F}, \delta) \in I$. By Condition (iii), we have that $\mathcal{P} \npreceq \mathcal{M}^{\omega} \mathcal{B}^{\omega}$. This implies, in particular, that there exists a unitary $u \in \mathcal{U}(\mathcal{P})$ such that $\left\|\mathrm{E}_{\mathcal{B}^{\omega}}\left(b^{*} u a\right)\right\|_{2}<\delta$ for all $a, b \in \mathcal{F}$. Since $p \mathcal{M} p$ is a finite von Neumann algebra, we may write $u=\left(u_{n}\right)^{\omega} \in \mathcal{U}(\mathcal{P})$ for some $\left(u_{n}\right)_{n} \in \ell^{\infty}(\mathbf{N}, p \mathcal{M} p)$ such that $u_{n} \in \mathcal{U}(p \mathcal{M} p)$ for all $n \in \mathbf{N}$. Observe that $\lim _{n \rightarrow \omega}\left\|u_{n} x-x u_{n}\right\|_{2}=\|u x-x u\|_{2}=0$, $\left\|\mathrm{E}_{\mathcal{B}^{\omega}}\left(b^{*} u a\right)\right\|_{2}=\lim _{n \rightarrow \omega}\left\|\mathrm{E}_{\mathcal{B}}\left(b^{*} u_{n} a\right)\right\|_{2}<\delta$ for all $a, b \in \mathcal{F}$ and $\left\|u-\theta_{t}^{\omega}(u)\right\|_{2}=\lim _{n \rightarrow \omega} \| u_{n}-$ $\theta_{t}\left(u_{n}\right) \|_{2}$. Thus, there exists $n \in \mathbf{N}$ large enough such that $v_{i}:=u_{n} \in \mathcal{U}(p \mathcal{M} p)$ satisfies the following properties:

- $\left\|v_{i} x-x v_{i}\right\|_{2} \leq \delta$,

- $\left\|\mathrm{E}_{\mathcal{B}}\left(b^{*} v_{i} a\right)\right\|_{2} \leq \delta$ for all $a, b \in \mathcal{F}$ and

- $\left\|v_{i}-\theta_{t}\left(v_{i}\right)\right\|_{2} \leq\left\|u-\theta_{t}^{\omega}(u)\right\|_{2}+\frac{\varepsilon^{2}}{16} \leq \frac{\varepsilon^{2}}{8}$. 
Put $\delta_{t}(y)=\theta_{t}(y)-\mathrm{E}_{\mathcal{M}}\left(\theta_{t}(y)\right) \in \widetilde{\mathcal{M}} \ominus \mathcal{M}$ for all $y \in p \mathcal{M} p$. For all $i \in I$, we have

$$
\begin{aligned}
\left\|\delta_{t}(x)\right\|_{2}^{2}=\left\langle\delta_{t}(x), \delta_{t}(x)\right\rangle & \leq\left|\left\langle\delta_{t}\left(v_{i} x v_{i}^{*}\right), \delta_{t}(x)\right\rangle\right|+\left\|v_{i} x v_{i}^{*}-x\right\|_{2} \\
& \leq\left|\left\langle v_{i} \delta_{t}(x) v_{i}^{*}, \delta_{t}(x)\right\rangle\right|+\left\|v_{i} x v_{i}^{*}-x\right\|_{2}+2\left\|v_{i}-\theta_{t}\left(v_{i}\right)\right\|_{2} \\
& \leq\left|\left\langle v_{i} \delta_{t}(x) v_{i}^{*}, \delta_{t}(x)\right\rangle\right|+\left\|v_{i} x v_{i}^{*}-x\right\|_{2}+\frac{\varepsilon^{2}}{4} .
\end{aligned}
$$

Since $\lim _{i}\left\|\mathrm{E}_{\mathcal{B}}\left(b^{*} v_{i} a\right)\right\|_{2}=0$ for all $a, b \in p \mathcal{M}$, we have $\lim _{i}\left\|\mathrm{E}_{\mathcal{M}}\left(d^{*} v_{i} c\right)\right\|_{2}=0$ for all $c, d \in$ $p(\widetilde{\mathcal{M}} \ominus \mathcal{M})$ by Condition (iv). In particular, using Cauchy-Schwarz inequality in $\mathrm{L}^{2}(\widetilde{\mathcal{M}})$, we have

$$
\begin{aligned}
\limsup _{i}\left|\left\langle v_{i} \delta_{t}(x) v_{i}^{*}, \delta_{t}(x)\right\rangle\right| & =\limsup _{i}\left|\left\langle\delta_{t}(x)^{*} v_{i} \delta_{t}(x), v_{i}\right\rangle\right| \\
& =\limsup _{i}\left|\left\langle\mathrm{E}_{\mathcal{M}}\left(\delta_{t}(x)^{*} v_{i} \delta_{t}(x)\right), v_{i}\right\rangle\right| \\
& \leq \limsup _{i}\left\|\mathrm{E}_{\mathcal{M}}\left(\left(p \delta_{t}(x)\right)^{*} v_{i} p \delta_{t}(x)\right)\right\|_{2}\left\|v_{i}\right\|_{2} \\
& =0 .
\end{aligned}
$$

Combining (4.1) and (4.2) with the first property of the net $\left(v_{i}\right)_{i \in I}$ and the transversality property of the malleable deformation $\left(\theta_{t}\right)$, we obtain

$$
\left\|x-\theta_{2 t}(x)\right\|_{2} \leq 2\left\|\delta_{t}(x)\right\|_{2} \leq \varepsilon .
$$

Since the above inequality holds for all $x \in \operatorname{Ball}(\mathcal{Q})$ and all $t \in\left[-t_{0}, t_{0}\right]$, we have obtained that the map $\mathbf{R} \rightarrow \operatorname{Aut}(\widetilde{\mathcal{M}}): t \mapsto \theta_{t}$ converges uniformly on $\operatorname{Ball}(\mathcal{Q})$ in $\|\cdot\|_{2}$ as $t \rightarrow 0$. This finishes the proof of Theorem 4.1.

As a corollary to Theorem 4.1, we obtain the following 'location' result for subalgebras in semifinite amalgamated free product von Neumann algebras. For each $i \in\{1,2\}$, let $\mathcal{B} \subset \mathcal{M}_{i}$ be an inclusion of $\sigma$-finite semifinite von Neumann algebras with expectation $\mathrm{E}_{i}: \mathcal{M}_{i} \rightarrow \mathcal{B}$. Let $\operatorname{Tr}_{\mathcal{B}}$ be a faithful normal semifinite trace such that the weight $\operatorname{Tr}_{\mathcal{B}} \circ \mathrm{E}_{i}$ is tracial on $\mathcal{M}_{i}$ for all $i \in\{1,2\}$. Then the amalgamated free product $(\mathcal{M}, \mathrm{E})=\left(\mathcal{M}_{1}, \mathrm{E}_{1}\right) *_{\mathcal{B}}\left(\mathcal{M}_{1}, \mathrm{E}_{1}\right)$ is semifinite and the weight $\operatorname{Tr}=\operatorname{Tr}_{\mathcal{B}} \circ \mathrm{E}$ is tracial on $\mathcal{M}$ as remarked in Section 2

Corollary 4.2. Keep the same notation as above. Assume moreover that $\mathcal{B}$ is amenable. Let $p \in \mathcal{M}$ be any nonzero finite trace projection and $\mathcal{Q} \subset p \mathcal{M} p$ any von Neumann subalgebra with no amenable direct summand such that $\mathcal{Q}^{\prime} \cap(p \mathcal{M} p)^{\omega} \npreceq \mathcal{M}^{\omega} \mathcal{B}^{\omega}$ for some nonprincipal ultrafilter $\omega \in \beta(\mathbf{N}) \backslash \mathbf{N}$.

Then for every nonzero projection $z \in \mathcal{Q}^{\prime} \cap p \mathcal{M} p$, there exists $i \in\{1,2\}$ such that $\mathcal{Q} z \preceq \mathcal{M} \mathcal{M}_{i}$.

Proof. Put

$$
\widetilde{\mathcal{M}}=\mathcal{M} *_{\mathcal{B}}\left(\mathcal{B} \bar{\otimes} \mathrm{L}\left(\mathbf{F}_{2}\right)\right)
$$

and consider the trace preserving free malleable deformation $\left(\theta_{t}\right)$ from [IPP05, Section 2] on $\widetilde{\mathcal{M}}$ (see [BHR12, Section 3] for further details).

We now check that we can apply Theorem 4.1 to our situation.

(i) Since $\mathcal{B}$ is amenable, the $p \mathcal{M} p-p \mathcal{M} p$-bimodule $\mathrm{L}^{2}(p \widetilde{\mathcal{M}} p) \ominus \mathrm{L}^{2}(p \mathcal{M} p)$ is weakly contained in the coarse $p \mathcal{M} p-p \mathcal{M} p$-bimodule $\mathrm{L}^{2}(p \mathcal{M} p) \otimes \mathrm{L}^{2}(p \mathcal{M} p)$ (see e.g. the proof of [CH08, Proposition 3.1]).

(ii) By assumption, the von Neumann algebra $\mathcal{Q}$ has no amenable direct summand.

(iii) By assumption, we have $\mathcal{Q}^{\prime} \cap(p \mathcal{M} p)^{\omega} \npreceq \mathcal{M}^{\omega} \mathcal{B}^{\omega}$ for some nonprincipal ultrafilter $\omega \in$ $\beta(\mathbf{N}) \backslash \mathbf{N}$. 
(iv) Let $\left(v_{i}\right)_{i \in I}$ be any net of unitaries in $\mathcal{U}(p \mathcal{M} p)$ such that $\lim _{i}\left\|\mathrm{E}_{\mathcal{B}}\left(b^{*} v_{i} a\right)\right\|_{2}=0$ for all $a, b \in p \mathcal{M}$. Since $\widetilde{\mathcal{M}}=\mathcal{M} *_{\mathcal{B}}\left(\mathcal{B} \otimes \mathrm{L}\left(\mathbf{F}_{2}\right)\right)$, the proof of [BHR12, Theorem 2.5, Claim] implies that $\lim _{i}\left\|\mathrm{E}_{\mathcal{M}}\left(d^{*} v_{i} c\right)\right\|_{2}=0$ for all $c, d \in p(\widetilde{\mathcal{M}} \ominus \mathcal{M})$.

Therefore, Theorem 4.1 implies that the map $\mathbf{R} \rightarrow \operatorname{Aut}(\widetilde{\mathcal{M}}): t \mapsto \theta_{t}$ converges uniformly on $\operatorname{Ball}(\mathcal{Q})$ in $\|\cdot\|_{2}$ as $t \rightarrow 0$. Fix now any nonzero projection $z \in \mathcal{Q}^{\prime} \cap p \mathcal{M} p$. We still have that the map $\mathbf{R} \rightarrow \operatorname{Aut}(\widetilde{\mathcal{M}}): t \mapsto \theta_{t}$ converges uniformly on Ball( $\left.\mathcal{Q} z\right)$ in $\|\cdot\|_{2}$ as $t \rightarrow 0$. Then, [BHR12, Theorem 3.3] implies that there exists $i \in\{1,2\}$ such that $\mathcal{Q} z \preceq \mathcal{M} \mathcal{M}_{i}$.

Proof of Theorem $\mathrm{A}$. Theorem $\mathrm{A}$ will be a consequence of the following optimal result that generalizes [Ho14, Theorem D] to arbitrary free product von Neumann algebras.

Theorem 4.3. For each $i \in\{1,2\}$, let $\left(M_{i}, \varphi_{i}\right)$ be any $\sigma$-finite von Neumann algebra endowed with a faithful normal state. Denote by $(M, \varphi)=\left(M_{1}, \varphi_{1}\right) *\left(M_{2}, \varphi_{2}\right)$ the free product. Let $Q \subset M$ be any von Neumann subalgebra with separable predual and with expectation such that $Q \cap M_{1}$ is diffuse and with expectation. Let $\omega \in \beta(\mathbf{N}) \backslash \mathbf{N}$ be any nonprincipal ultrafilter on $\mathbf{N}$.

Denote by $z \in \mathcal{Z}\left(Q^{\prime} \cap M^{\omega}\right)$ the unique central projection such that $\left(Q^{\prime} \cap M^{\omega}\right) z$ is diffuse and $\left(Q^{\prime} \cap M^{\omega}\right) z^{\perp}$ is atomic. Then the following conditions hold:

- $z \in \mathcal{Z}\left(Q^{\prime} \cap M\right)=\mathcal{Z}\left(Q^{\prime} \cap M_{1}\right)$,

- $Q z \subset z M_{1} z$ and

- $\left(Q^{\prime} \cap M^{\omega}\right) z^{\perp}=\left(Q^{\prime} \cap M\right) z^{\perp}=\left(Q^{\prime} \cap M_{1}\right) z^{\perp}$.

Throughout the rest of this section, let $(M, \varphi)=\left(M_{1}, \varphi_{1}\right) *\left(M_{2}, \varphi_{2}\right)$ be as in Theorem 4.3. Observe that $M_{1}$ is diffuse by assumption. Proposition 2.7 (1) implies that $\left(M_{1}\right)^{\prime} \cap M \subset M_{1}$. Therefore, there exists a unique faithful normal conditional expectation $\mathrm{E}_{M_{1}}: M \rightarrow M_{1}$ by Co72, Théorème 1.5.5]. We fix a nonprincipal ultrafilter $\omega \in \beta(\mathbf{N}) \backslash \mathbf{N}$.

For Lemmas 4.4 and 4.5 below, we moreover fix a faithful state $\psi \in M_{*}$ such that $\psi=\psi \circ \mathrm{E}_{M_{1}}$. Whenever $q \in M^{\psi}$ is a nonzero projection, put $\psi_{q}=\frac{\psi(q \cdot q)}{\psi(q)} \in(q M q)_{*}$.

Lemma 4.4. Let $q \in\left(M_{1}\right)^{\psi}$ be any nonzero projection and $Q \subset q M q$ any non type I subfactor with separable predual that is amenable and globally invariant under the modular automorphism group $\sigma^{\psi_{q}}$ and such that $Q \cap q M_{1} q$ is diffuse. Then $Q \subset q M_{1} q$.

Proof. The proof of Lemma 4.4 is inspired by the one of [Ho12b, Theorem 8.1]. We will consider successively the cases when $Q$ is of type $\mathrm{II}_{1}$, of type $\mathrm{II}_{\infty}$ and of type III.

Case type $\mathrm{II}_{1}$. Assume that $q \in\left(M_{1}\right)^{\psi}$ is any nonzero projection and $Q \subset q M q$ is any type $\mathrm{II}_{1}$ subfactor with separable predual that is amenable and globally invariant under the modular automorphism group $\sigma^{\psi_{q}}$ and such that $Q \cap q M_{1} q$ is diffuse. Then we have $Q \subset q M_{1} q$.

We start by showing the following claim.

Claim. For any nonzero projection $z \in \mathcal{Z}\left(Q^{\prime} \cap q M q\right)$, we have $Q z \preceq_{M} M_{1}$.

Proof of the Claim. By contradiction, assume that there exists a nonzero projection $z \in \mathcal{Z}\left(Q^{\prime} \cap\right.$ $q M q$ ) such that $Q z \npreceq M_{M} M_{1}$. Since $Q^{\prime} \cap q M q \subset q M_{1} q$ by Proposition 2.7 (1) and $Q^{\prime} \cap q M q \subset$ $q M_{1} q$ is globally invariant under the modular automorphism group $\sigma^{\psi_{q}}$, we have $z \in\left(M_{1}\right)^{\psi}$. Write $Q=\bigvee_{n \in \mathbf{N}} Q_{n}$ where $\left(Q_{n}\right)_{n}$ is an increasing sequence of finite dimensional subfactors of $Q$ of the form $Q_{n} \cong \mathbf{M}_{2^{n}}(\mathbf{C})$. Since the inclusion

$$
\left(Q_{n}^{\prime} \cap Q\right) z \subset Q z \cong Q_{n}^{\prime} \cap Q \subset Q
$$

(n.b. $Q$ is a factor) has finite index, Lemma 2.3 implies that $\left(Q_{n}^{\prime} \cap Q\right) z \npreceq M_{M} M_{1}$ for all $n \in \mathbf{N}$. 
Then for every $n \in \mathbf{N}$, choose a unitary $u_{n} \in \mathcal{U}\left(\left(Q_{n}^{\prime} \cap Q\right) z\right)$ such that $\left\|\mathrm{E}_{M_{1}}\left(u_{n}\right)\right\|_{\psi} \leq \frac{1}{n+1}$. Since $Q z$ is finite with expectation, we have $\left(u_{n}\right)_{n} \in \mathcal{M}^{\omega}(z M z)$ and hence we may define $u=\left(u_{n}\right)^{\omega} \in(z M z)^{\omega}=z M^{\omega} z \subset M^{\omega}$. We then have $u \in(Q z)^{\prime} \cap(Q z)^{\omega}$ and $\mathrm{E}_{M_{1}^{\omega}}(u)=0$ since

$$
\left\|\mathrm{E}_{M_{1}^{\omega}}(u)\right\|_{\psi^{\omega}}=\lim _{n \rightarrow \omega}\left\|\mathrm{E}_{M_{1}}\left(u_{n}\right)\right\|_{\psi}=0 .
$$

Observe that $\left(Q z \cap z M_{1} z\right) \oplus z^{\perp} M_{1} z^{\perp} \subset M_{1}$ is a diffuse von Neumann subalgebra that is globally invariant under the modular automorphism group $\sigma^{\psi}$. Since $u \in(Q z)^{\prime} \cap(Q z)^{\omega}$, we have $u \in\left(\left(Q z \cap z M_{1} z\right) \oplus z^{\perp} M_{1} z^{\perp}\right)^{\prime} \cap M^{\omega}$. For all $n \in \mathbf{N}$, since we moreover have $u u_{n}=u_{n} u$ and $u^{*} u=z$, Theorem 3.1 implies that

$$
\begin{aligned}
\left\|\mathrm{E}_{M_{1}}\left(u_{n}\right) u-u \mathrm{E}_{M_{1}}\left(u_{n}\right)\right\|_{\psi^{\omega}} & =\left\|\left(\mathrm{E}_{M_{1}}\left(u_{n}\right)-u_{n}\right) u+u\left(u_{n}-\mathrm{E}_{M_{1}}\left(u_{n}\right)\right)\right\|_{\psi^{\omega}} \\
& \geq\left\|u\left(u_{n}-\mathrm{E}_{M_{1}}\left(u_{n}\right)\right)\right\|_{\psi^{\omega}} \quad \text { (use Theorem 3.1 here) } \\
& \geq\|z\|_{\psi}-\left\|\mathrm{E}_{M_{1}}\left(u_{n}\right)\right\|_{\psi} .
\end{aligned}
$$

Observe that $\lim _{n \rightarrow \infty} \mathrm{E}_{M_{1}}\left(u_{n}\right)=0 \sigma$-strongly. By taking the limit as $n \rightarrow \infty$ in the above inequality, we obtain $z=0$, a contradiction. This finishes the proof of the Claim.

The set $\mathfrak{R}$ of projections $r \in Q^{\prime} \cap q M q=Q^{\prime} \cap q M_{1} q$ (by Proposition 2.7 (1)) such that $Q r \subset r M_{1} r$ attains its maximum in a unique projection $z$ that belongs to $\mathcal{Z}\left(Q^{\prime} \cap q M q\right)=$ $\mathcal{Z}\left(Q^{\prime} \cap q M_{1} q\right)$. (In fact, $\mathfrak{R}$ is invariant under the adjoint action of $\mathcal{U}\left(Q^{\prime} \cap q M_{1} q\right)$, and $z:=$ $\bigvee_{r \in \mathfrak{R}} r \in \mathcal{Z}\left(Q^{\prime} \cap q M_{1} q\right)$ must satisfy $x z=\mathrm{E}_{M_{1}}(x) z=z \mathrm{E}_{M_{1}}(x) z$ for all $x \in Q$.) Assume by contradiction that $z \neq q$. Put $z^{\perp}:=q-z \in \mathcal{Z}\left(Q^{\prime} \cap q M q\right)$. By assumption, we have $z^{\perp} \neq 0$.

By the previous Claim, we have that $Q z^{\perp} \preceq_{M} M_{1}$. Then there exist $n \geq 1$, a projection $p \in \mathbf{M}_{n}\left(M_{1}\right)$, a nonzero partial isometry $v \in\left(z^{\perp} M \otimes \mathbf{M}_{1, n}(\mathbf{C})\right) p$ and a unital normal *homomorphism $\pi: Q z^{\perp} \rightarrow p \mathbf{M}_{n}\left(M_{1}\right) p$ such that the inclusion $\pi\left(Q z^{\perp}\right) \subset p \mathbf{M}_{n}\left(M_{1}\right) p$ is with expectation (see Theorem 2.2 due to the first named author and Isono [HI15] for this important property) and $a v=v \pi(a)$ for all $a \in Q z^{\perp}$. By Proposition 2.7 (1), we obtain that $v \in$ $\left(z^{\perp} M_{1} \otimes \mathbf{M}_{1, n}(\mathbf{C})\right) p$ and hence $v v^{*} \in z^{\perp}\left(Q^{\prime} \cap q M q\right) z^{\perp}=z^{\perp}\left(Q^{\prime} \cap q M_{1} q\right) z^{\perp}$ and $Q z^{\perp} v v^{*} \subset$ $v v^{*} z^{\perp} M_{1} z^{\perp} v v^{*}$. Since $v v^{*} \leq z^{\perp}, v v^{*} \neq 0$ and $Q\left(z+v v^{*}\right) \subset\left(z+v v^{*}\right) M_{1}\left(z+v v^{*}\right)$, this contradicts the maximality of $z \in Q^{\prime} \cap q M_{1} q$ and finishes the proof in the case when $Q$ is of type $\mathrm{II}_{1}$.

Case type $\mathrm{II}_{\infty}$. Assume that $q \in\left(M_{1}\right)^{\psi}$ is any nonzero projection and $Q \subset q M q$ is any type $\mathrm{II}_{\infty}$ subfactor with separable predual that is amenable and globally invariant under the modular automorphism group $\sigma^{\psi_{q}}$ and such that $Q \cap q M_{1} q$ is diffuse. Then we have $Q \subset q M_{1} q$.

Choose a faithful normal semifinite trace $\operatorname{Tr}$ on $Q$ and write $\psi_{q}=\operatorname{Tr}(T \cdot)$ for some positive nonsingular operator $T \in \mathrm{L}^{1}(Q, \mathrm{Tr})_{+}$(see e.g. [Ta03, Corollary VIII.3.6, Lemma IX.2.12]). Define the abelian von Neumann subalgebra $B=\left\{T^{\mathrm{i} t}: t \in \mathbf{R}\right\}^{\prime \prime} \subset Q$. Since $\sigma_{t}^{\psi_{q}}=\operatorname{Ad}\left(T^{\mathrm{i} t}\right)$ for all $t \in \mathbf{R}$, we have $Q^{\psi_{q}}=B^{\prime} \cap Q$. Observe that since the inclusion $Q \cap q M_{1} q \subset Q$ is globally invariant under the modular automorphism group $\sigma^{\psi_{q}}$, the diffuse von Neumann subalgebra $Q \cap q M_{1} q \subset Q$ is also semifinite and hence its centralizer $\left(Q \cap q M_{1} q\right)^{\psi_{q}}$ is diffuse (see e.g. Ue98b, Lemma 11]). By Proposition 2.7 (1) and since $B$ is abelian, we have

$$
B \subset\left(Q^{\psi_{q}}\right)^{\prime} \cap Q^{\psi_{q}} \subset\left(\left(Q \cap q M_{1} q\right)^{\psi_{q}}\right)^{\prime} \cap Q^{\psi_{q}} \subset Q^{\psi_{q}} \cap q M_{1} q=\left(Q \cap q M_{1} q\right)^{\psi_{q}} .
$$

For every $k \in \mathbf{N}$, we denote by $q_{k}$ the spectral projection of $T$ for the interval $\left[\frac{1}{k},+\infty\right)$. Then all $q_{k}$ are Tr-finite projections in $B$ such that $q_{k} \rightarrow q$, the unit of $Q, \sigma$-strongly as $k \rightarrow \infty$. Since $q_{k} \in\left(Q \cap q M_{1} q\right)^{\psi_{q}}$, the type $\mathrm{II}_{1}$ subfactor $q_{k} Q q_{k} \subset q_{k} M q_{k}$ is amenable and globally invariant under the modular automorphism group $\sigma^{\psi_{q_{k}}}$ and $q_{k} Q q_{k} \cap q_{k} M_{1} q_{k}=q_{k}\left(Q \cap q M_{1} q\right) q_{k}$ is diffuse. We may then apply the result obtained in the first case to the $\mathrm{II}_{1}$ subfactor $q_{k} Q q_{k} \subset q_{k} M q_{k}$ 
and we have that $q_{k} Q q_{k} \subset q_{k} M_{1} q_{k}$ for all $k \in \mathbf{N}$. Since $q_{k} \rightarrow q \sigma$-strongly as $k \rightarrow \infty$, we obtain $Q \subset q M_{1} q$. This finishes the proof in the case when $Q$ is of type $\mathrm{II}_{\infty}$.

Case type III. Assume that $q \in\left(M_{1}\right)^{\psi}$ is any nonzero projection and $Q \subset q M q$ is any type III subfactor with separable predual that is amenable and globally invariant under the modular automorphism group $\sigma^{\psi_{q}}$ and such that $Q \cap q M_{1} q$ is diffuse. Then we have $Q \subset q M_{1} q$.

By combining results on the classification theory of amenable factors [Co72, Co75, Ha85] together with [FM75, Kr75, there exists a hyperfinite ergodic nonsingular equivalence relation $\mathcal{R}$ defined on a standard probability space $(X, \mu)$ such that $Q=\mathrm{L}(\mathcal{R})$. Put $A=\mathrm{L}^{\infty}(X)$ and denote by $\mathrm{E}_{A}: Q \rightarrow A$ the unique faithful normal conditional expectation. Denote by $\mathrm{E}_{Q}: q M q \rightarrow Q$ the unique $\psi_{q}$-preserving conditional expectation. Choose any faithful state $\tau_{A} \in A_{*}$ and put $\phi=\tau_{A} \circ \mathrm{E}_{A} \circ \mathrm{E}_{Q} \in(q M q)_{*}$. Observe that $A \subset(q M q)^{\phi}$ and $Q$ is globally invariant under the modular automorphism group $\sigma^{\phi}$.

Let $\left(\mathcal{R}_{n}\right)_{n \in \mathbf{N}}$ be an increasing sequence of finite subequivalence relations of $\mathcal{R}$ such that $\mathcal{R}=$ $\bigvee_{n \in \mathbf{N}} \mathcal{R}_{n}$. Put $Q_{n}=\mathrm{L}\left(\mathcal{R}_{n}\right)$ for all $n \in \mathbf{N}$ so that $Q=\bigvee_{n \in \mathbf{N}} Q_{n}$. Note that $A \subset Q_{n}$ is still a Cartan subalgebra and $Q_{n}$ is globally invariant under the modular automorphism group $\sigma^{\phi}$ for all $n \in \mathbf{N}$ because $\left.\phi\right|_{Q}=\tau_{A} \circ \mathrm{E}_{A}$. Observe that since $\mathcal{R}_{n}$ is finite, that is, $\mathcal{R}_{n}$ has finite orbits almost everywhere, $Q_{n}$ is a countable direct sum of finite type I von Neumann algebras. Therefore using [Ka82, Corollary 3.19], up to conjugating by a unitary in $\mathcal{U}\left(Q_{n}\right)$, the inclusion $A \subset Q_{n}$ is of the following form:

$$
\left(A \subset Q_{n}\right) \cong\left(\bigoplus_{k \in \mathbf{N}} \mathcal{Z}_{n}^{(k)} \otimes \mathbf{C}^{\oplus k} \subset \bigoplus_{k \in \mathbf{N}} \mathcal{Z}_{n}^{(k)} \otimes \mathbf{M}_{k}(\mathbf{C})\right),
$$

where $\mathcal{Z}_{n}^{(k)}$ is a diffuse abelian von Neumann algebra for all $n, k \in \mathbf{N}$.

Claim. For any nonzero projection $z \in \mathcal{Z}\left(Q^{\prime} \cap q M q\right)$, we have $A z \preceq_{M} M_{1}$.

Proof of the Claim. By contradiction, assume that there exists a nonzero projection $z \in \mathcal{Z}\left(Q^{\prime} \cap\right.$ $q M q)$ such that $A z \npreceq M M_{1}$. Observe that $z \in(q M q)^{\phi} \cap\left(M_{1}\right)^{\psi}$. Using the structure of the inclusion $A \subset Q_{n}$ as in (4.3), we see that the inclusion $Q_{n}^{\prime} \cap A \subset A$ is of the form:

$$
\left(Q_{n}^{\prime} \cap A \subset A\right) \cong\left(\bigoplus_{k \in \mathbf{N}} \mathcal{Z}_{n}^{(k)} \otimes \mathbf{C} 1 \subset \bigoplus_{k \in \mathbf{N}} \mathcal{Z}_{n}^{(k)} \otimes \mathbf{C}^{\oplus k}\right) .
$$

Using (4.4), it follows that the inclusion

$$
\left(Q_{n}^{\prime} \cap A\right) z \subset A z \cong Q_{n}^{\prime} \cap A \subset A
$$

has essentially finite index and Lemma 2.3 implies that $\left(Q_{n}^{\prime} \cap A\right) z \npreceq M_{M} M_{1}$ for all $n \in \mathbf{N}$. (Remark that this can easily be confirmed directly, since $Q_{n}^{\prime} \cap A \subset A$ are commutative.)

Then for every $n \in \mathbf{N}$, choose a unitary $u_{n} \in \mathcal{U}\left(\left(Q_{n}^{\prime} \cap A\right) z\right)$ such that $\left\|\mathrm{E}_{M_{1}}\left(u_{n}\right)\right\|_{\psi} \leq \frac{1}{n+1}$. Since $u_{n} \in(z M z)^{\phi_{z}}$ for all $n \in \mathbf{N}$, we may define $u=\left(u_{n}\right)^{\omega} \in(z M z)^{\omega}=z M^{\omega} z \subset M^{\omega}$. We then have $u \in(Q z)^{\prime} \cap(A z)^{\omega}$ and $\mathrm{E}_{M_{1}^{\omega}}(u)=0$. Observe that $u \in\left(\left(Q z \cap z M_{1} z\right) \oplus z^{\perp} M_{1} z^{\perp}\right)^{\prime} \cap M^{\omega}$. For all $n \in \mathbf{N}$, Theorem 3.1 implies, as in Case type $\mathrm{II}_{1}$, that

$$
\left\|\mathrm{E}_{M_{1}}\left(u_{n}\right) u-u \mathrm{E}_{M_{1}}\left(u_{n}\right)\right\|_{\psi^{\omega}} \geq\|z\|_{\psi}-\left\|\mathrm{E}_{M_{1}}\left(u_{n}\right)\right\|_{\psi}
$$

and hence $z=0$, a contradiction. This finishes the proof of the Claim.

The set of projections $r \in Q^{\prime} \cap q M q=Q^{\prime} \cap q M_{1} q$ (by Proposition 2.7 (1)) such that $Q r \subset r M_{1} r$ attains its maximum in a unique projection $z$ that belongs to $\mathcal{Z}\left(Q^{\prime} \cap q M q\right)=\mathcal{Z}\left(Q^{\prime} \cap q M_{1} q\right)$ (see Case type $\left.\mathrm{II}_{1}\right)$. Assume by contradiction that $z \neq q$. Put $z^{\perp}:=q-z \in \mathcal{Z}\left(Q^{\prime} \cap q M q\right)$. By assumption, we have $z^{\perp} \neq 0$ and moreover $z^{\perp} \in Q^{\prime} \cap q M q \subset A^{\prime} \cap q M q$. 
By the previous Claim, we have that $A z^{\perp} \preceq_{M} M_{1}$. Then there exist $n \geq 1$, a projection $p \in \mathbf{M}_{n}\left(M_{1}\right)$, a nonzero partial isometry $v \in\left(z^{\perp} M \otimes \mathbf{M}_{1, n}(\mathbf{C})\right) p$ and a unital normal *homomorphism $\pi: A z^{\perp} \rightarrow p \mathbf{M}_{n}\left(M_{1}\right) p$ such that the inclusion $\pi\left(A z^{\perp}\right) \subset p \mathbf{M}_{n}\left(M_{1}\right) p$ is with expectation (see Theorem 2.2) and $a v=v \pi(a)$ for all $a \in A z^{\perp}$. Since $z^{\perp} \in Q^{\prime} \cap q M q \subset A^{\prime} \cap q M q$, we may define the unital normal $*$-homomorphism $\iota: A \rightarrow A z^{\perp}: a \mapsto a z^{\perp}$. Then $\pi \circ \iota: A \rightarrow$ $p \mathbf{M}_{n}\left(M_{1}\right) p$ is unital normal $*$-homomorphism such that the inclusion $(\pi \circ \iota)(A) \subset p \mathbf{M}_{n}\left(M_{1}\right) p$ is with expectation and $a v=\iota(a) v=v \pi(\iota(a))=v(\pi \circ \iota)(a)$ for all $a \in A$.

Put $N=\mathcal{N}_{q M q}(A)^{\prime \prime}$ and observe that $Q \subset N$. Since $v^{*} v \in(\pi \circ \iota)(A)^{\prime} \cap p \mathbf{M}_{n}(M) p$ and since $(\pi \circ \iota)(A) \subset p \mathbf{M}_{n}\left(M_{1}\right) p$ is diffuse and with expectation, we have $v^{*} v \in(\pi \circ \iota)(A)^{\prime} \cap p \mathbf{M}_{n}\left(M_{1}\right) p$ by Proposition 2.7 (2) and hence we may assume that $p=v^{*} v$. Since the inclusion $A \subset N$ is regular, we moreover have $v^{*} N v \subset p \mathbf{M}_{n}\left(M_{1}\right) p$ by Proposition 2.7 (2).

We have $v v^{*} \in\left(A z^{\perp}\right)^{\prime} \cap z^{\perp} M z^{\perp}=z^{\perp}\left(A^{\prime} \cap q M q\right) z^{\perp} \subset z^{\perp} N z^{\perp}$. Since the inclusion $Q \subset N$ is with expectation (because so is $Q \subset q M q$ ) and since $Q$ is of type III, it follows that $N$ is also of type III (see [Ta02, Lemma V.2.29]) and so is $z^{\perp} N z^{\perp}$. If we denote by $r \in \mathcal{Z}\left(z^{\perp} N z^{\perp}\right)$ the central support in $z^{\perp} N z^{\perp}$ of the projection $v v^{*} \in z^{\perp} N z^{\perp}$, we have $v v^{*} \sim r$ in $z^{\perp} N z^{\perp}$. There exists a partial isometry $u \in z^{\perp} N z^{\perp}$ such that $u^{*} u=v v^{*}$ and $u u^{*}=r$. We have $(u v)^{*} N r(u v) \subset p \mathbf{M}_{n}\left(M_{1}\right) p$. So, up to replacing $v$ by $u v$, we may assume that $v^{*} z^{\perp} N z^{\perp} v \subset$ $p \mathbf{M}_{n}\left(M_{1}\right) p, v v^{*} \in \mathcal{Z}\left(z^{\perp} N z^{\perp}\right)$ and $p=v^{*} v$.

This implies that $z^{\perp} N z^{\perp} v \subset v p \mathbf{M}_{n}\left(M_{1}\right) p$ and hence $Q z^{\perp} v \subset v p \mathbf{M}_{n}\left(M_{1}\right) p$. This further implies that $\left(Q \cap q M_{1} q\right) z^{\perp} v \subset v p \mathbf{M}_{n}\left(M_{1}\right) p$. Since $p=v^{*} v, v v^{*} \in \mathcal{Z}\left(z^{\perp} N z^{\perp}\right)$ and $Q \subset N$, the mapping $\rho:\left(Q \cap q M_{1} q\right) z^{\perp} \rightarrow p \mathbf{M}_{n}\left(M_{1}\right) p: x \mapsto v^{*} x v$ defines a unital normal $*$-homomorphism such that $x v=v \rho(x)$ for all $x \in\left(Q \cap q M_{1} q\right) z^{\perp}$. Observe that $z \in M^{\psi}$ and hence $\left(Q \cap q M_{1} q\right) z^{\perp} \subset$ $z^{\perp} M z^{\perp}$ is with expectation. By Proposition 2.7 (1), we obtain that $v \in\left(z^{\perp} M_{1} \otimes \mathbf{M}_{1, n}(\mathbf{C})\right) p$ and hence $v v^{*} \in z^{\perp}\left(Q^{\prime} \cap q M q\right) z^{\perp}=z^{\perp}\left(Q^{\prime} \cap q M_{1} q\right) z^{\perp}$ and $Q z^{\perp} v v^{*} \subset v v^{*} z^{\perp} M_{1} z^{\perp} v v^{*}$. Since $v v^{*} \leq z^{\perp}, v v^{*} \neq 0$ and $Q\left(z+v v^{*}\right) \subset\left(z+v v^{*}\right) M_{1}\left(z+v v^{*}\right)$, this contradicts the maximality of $z \in \bar{Q}^{\prime} \cap q M_{1} q$ and finishes the proof in the case when $Q$ is of type III.

Since we have successively treated the cases when $Q$ is of type $\mathrm{II}_{1}$, of type $\mathrm{II}_{\infty}$ and of type III, this finishes the proof of Lemma 4.4.

Lemma 4.5. Let $q \in\left(M_{1}\right)^{\psi}$ be any nonzero projection and $Q \subset q M q$ any subfactor with separable predual that is not amenable and globally invariant under the modular automorphism group $\sigma^{\psi_{q}}$ and such that $Q \cap q M_{1} q$ and $Q^{\prime} \cap(q M q)^{\omega}$ are diffuse. Then $Q \subset q M_{1} q$.

Proof. The proof, inspired by the one of [Ho12b, Theorem E], relies on Connes-Takesaki's structure theory Co72, Ta03, and uses Corollary 4.2 .

The novel aspect of the proof consists in combining [AH12, Theorem 4.1] and [MT13, Theorem 2.10] in order to obtain the following canonical inclusions of semifinite von Neumann algebras with trace preserving conditional expectations:

$$
\mathrm{c}_{\phi}(M) \subset \mathrm{c}_{\phi^{\omega}}\left(M^{\omega}\right) \subset\left(\mathrm{c}_{\phi}(M)\right)^{\omega}
$$

with $\phi=\varphi$ or $\phi=\psi$. More precisely, if we denote by $\mathrm{E}_{\omega}^{\phi}:\left(\mathrm{c}_{\phi}(M)\right)^{\omega} \rightarrow \mathrm{c}_{\phi}(M)$ the canonical faithful normal conditional expectation and by $\operatorname{Tr}_{\phi}$ (resp. $\operatorname{Tr}_{\phi \omega}$ ) the canonical faithful normal semifinite trace on $\mathrm{c}_{\phi}(M)$ (resp. $\mathrm{c}_{\phi^{\omega}}\left(M^{\omega}\right)$ ), we have that $\operatorname{Tr}_{\phi} \circ \mathrm{E}_{\omega}^{\phi}$ is a faithful normal semifinite trace on $\left(\mathrm{c}_{\phi}(M)\right)^{\omega}$ and $\left.\left(\operatorname{Tr}_{\phi} \circ \mathrm{E}_{\omega}^{\phi}\right)\right|_{\mathrm{c}_{\phi} \omega\left(M^{\omega}\right)}=\operatorname{Tr}_{\phi^{\omega}}$. We will simply use the notation $\|\cdot\|_{2}$ for the $\mathrm{L}^{2}$-norm associated with any of the faithful normal semifinite traces considered above. We will use throughout the proof the identification $\mathrm{L}_{\phi}(\mathbf{R})=\mathrm{L}_{\phi^{\omega}}(\mathbf{R}) \subset \mathrm{c}_{\phi^{\omega}}\left(M^{\omega}\right)$.

Since $q \in M^{\psi}$ and $Q \subset q M q$ is globally invariant under the modular automorphism group $\sigma^{\psi_{q}}$, we may define $\mathrm{c}_{\psi_{q}}(Q)=Q \rtimes_{\sigma^{\psi_{q}}} \mathbf{R}$ and regard $\mathrm{c}_{\psi_{q}}(Q) \subset \pi_{\psi}(q) \mathrm{c}_{\psi}(M) \pi_{\psi}(q)$ naturally. Fix an 
arbitrary nonzero finite trace projection $r \in \mathrm{L}_{\psi}(\mathbf{R})$ and put $\mathcal{M}=\mathrm{c}_{\varphi}(M), p=\Pi_{\varphi, \psi}(r) \in \mathcal{M}$, $\mathcal{Q}=\Pi_{\varphi, \psi}\left(r \mathrm{c}_{\psi_{q}}(Q) r\right)$ and $\mathcal{P}=\mathcal{Q}^{\prime} \cap\left(p \pi_{\varphi}(q) \mathcal{M} \pi_{\varphi}(q) p\right)^{\omega}$. Observe that

$$
p \pi_{\varphi}(q)=\Pi_{\varphi, \psi}\left(r \pi_{\psi}(q)\right)=\Pi_{\varphi, \psi}\left(\pi_{\psi}(q) r\right)=\pi_{\varphi}(q) p
$$

defines a nonzero projection in $\mathcal{M}$ and is the unit of $\mathcal{Q}$.

Claim. We have $\mathcal{P} \npreceq \mathcal{M}^{\omega}\left(\mathrm{L}_{\varphi}(\mathbf{R})\right)^{\omega}$.

Proof of the Claim. The proof uses an idea of [Io12, Lemma 9.5]. By contradiction, assume that $\mathcal{P} \preceq_{\mathcal{M}^{\omega}}\left(\mathrm{L}_{\varphi}(\mathbf{R})\right)^{\omega}$. By [BHR12, Lemma 2.3], there exist $\delta>0$ and a finite subset $\mathcal{F} \subset p \pi_{\varphi}(q) \mathcal{M}^{\omega}$ such that

$$
\sum_{a, b \in \mathcal{F}}\left\|\mathrm{E}_{\left(\mathrm{L}_{\varphi}(\mathbf{R})\right)^{\omega}}\left(b^{*} u a\right)\right\|_{2}^{2}>\delta, \forall u \in \mathcal{U}(\mathcal{P}) .
$$

For each $a \in \mathcal{F}$, write $a=\left(a_{n}\right)^{\omega}$ with a fixed sequence $\left(a_{n}\right)_{n} \in p \pi_{\varphi}(q) \mathcal{M}^{\omega}(\mathcal{M})$.

We next show that there exists $n \in \mathbf{N}$ such that

$$
\sum_{a, b \in \mathcal{F}}\left\|\mathrm{E}_{\left(\mathrm{L}_{\varphi}(\mathbf{R})\right)^{\omega}}\left(b_{n}^{*} u a_{n}\right)\right\|_{2}^{2} \geq \delta, \forall u \in \mathcal{U}(\mathcal{P}) .
$$

Assume by contradiction that this is not the case. Then for each $n \in \mathbf{N}$, there exists $u_{n} \in \mathcal{U}(\mathcal{P})$ such that

$$
\sum_{a, b \in \mathcal{F}}\left\|\mathrm{E}_{\left(\mathrm{L}_{\varphi}(\mathbf{R})\right)^{\omega}}\left(b_{n}^{*} u_{n} a_{n}\right)\right\|_{2}^{2}<\delta .
$$

Since $p \pi_{\varphi}(q) \mathcal{M} \pi_{\varphi}(q) p$ is a finite von Neumann algebra, we may write $u_{n}=\left(u_{m}^{(n)}\right)^{\omega}$ for some sequence $\left(u_{m}^{(n)}\right)_{m} \in \ell^{\infty}\left(\mathbf{N}, p \pi_{\varphi}(q) \mathcal{M} \pi_{\varphi}(q) p\right)$ such that $u_{m}^{(n)} \in \mathcal{U}\left(p \pi_{\varphi}(q) \mathcal{M} \pi_{\varphi}(q) p\right)$ for all $m \in \mathbf{N}$. Then we have

$$
\lim _{m \rightarrow \omega} \sum_{a, b \in \mathcal{F}}\left\|\mathrm{E}_{\mathrm{L}_{\varphi}(\mathbf{R})}\left(b_{n}^{*} u_{m}^{(n)} a_{n}\right)\right\|_{2}^{2}<\delta .
$$

Fix a $\|\cdot\|_{2}$-dense countable subset $\left\{y_{n}: n \in \mathbf{N}\right\} \subset \mathcal{Q}$. Since $\lim _{m \rightarrow \omega}\left\|y_{j} u_{m}^{(n)}-u_{m}^{(n)} y_{j}\right\|_{2}=$ $\left\|y_{j} u_{n}-u_{n} y_{j}\right\|_{2}=0$ for all $n \in \mathbf{N}$ and all $0 \leq j \leq n$, we may choose $m_{n} \in \mathbf{N}$ large enough so that $v_{n}:=u_{m_{n}}^{(n)} \in \mathcal{U}\left(p \pi_{\varphi}(q) \mathcal{M} \pi_{\varphi}(q) p\right)$ satisfies $\left\|y_{j} v_{n}-v_{n} y_{j}\right\|_{2} \leq \frac{1}{n+1}$ for all $0 \leq j \leq n$ and $\sum_{a, b \in \mathcal{F}}\left\|\mathrm{E}_{\mathrm{L}_{\varphi_{q}}(\mathbf{R})}\left(b_{n}^{*} v_{n} a_{n}\right)\right\|_{2}^{2} \leq \delta$. Since $p \pi_{\varphi}(q) \mathcal{M} \pi_{\varphi}(q) p$ is finite, we may define $v:=\left(v_{n}\right)^{\omega} \in$ $\left(p \pi_{\varphi}(q) \mathcal{M} \pi_{\varphi}(q) p\right)^{\omega}$. We moreover have $v \in \mathcal{U}(\mathcal{P})$ and

$$
\sum_{a, b \in \mathcal{F}}\left\|\mathrm{E}_{\left(\mathrm{L}_{\varphi}(\mathbf{R})\right)^{\omega}}\left(b^{*} v a\right)\right\|_{2}^{2}=\lim _{n \rightarrow \omega} \sum_{a, b \in \mathcal{F}}\left\|\mathrm{E}_{\mathrm{L}_{\varphi}(\mathbf{R})}\left(b_{n}^{*} v_{n} a_{n}\right)\right\|_{2}^{2} \leq \delta .
$$

Equations (4.5) and (4.7) give a contradiction. This shows that Equation (4.6) holds. Therefore, up to replacing the finite subset $\mathcal{F} \subset p \pi_{\varphi}(q) \mathcal{M}^{\omega}$ by $\left\{a_{n}: a \in \mathcal{F}\right\} \subset p \pi_{\varphi}(q) \mathcal{M}$, we may assume that $\mathcal{F} \subset p \pi_{\varphi}(q) \mathcal{M}$ in Equation (4.5).

Since $Q^{\prime} \cap(q M q)^{\omega}$ is diffuse and $Q$ is globally invariant under the modular automorphism group $\sigma^{\psi_{q}}$, we know that $Q^{\prime} \cap\left((q M q)^{\omega}\right)^{\psi_{q}^{\omega}}$ is diffuse by [HR14, Theorem 2.3]. We may then choose a sequence $\left(u_{n}\right)_{n} \in \mathcal{M}^{\omega}(q M q)$ such that $\left(u_{n}\right)^{\omega} \in \mathcal{U}\left(Q^{\prime} \cap\left((q M q)^{\omega}\right)^{\psi_{q}^{\omega}}\right)$ and $\lim _{n \rightarrow \infty} u_{n}=0$ $\sigma$-weakly (see the first and second paragraphs in the proof of [HR14, Theorem A]). Observe that $\left(p \pi_{\varphi}\left(u_{n}\right) p\right)_{n} \in \ell^{\infty}\left(\mathbf{N}, p \pi_{\varphi}(q) \mathcal{M} \pi_{\varphi}(q) p\right)$ and

$$
\begin{aligned}
\pi_{\varphi^{\omega}}\left(\left(u_{n}\right)^{\omega}\right) p & =\Pi_{\varphi^{\omega}, \psi^{\omega}}\left(\pi_{\psi^{\omega}}\left(\left(u_{n}\right)^{\omega}\right) r\right) \\
& =\Pi_{\varphi^{\omega}, \psi^{\omega}}\left(r \pi_{\psi^{\omega}}\left(\left(u_{n}\right)^{\omega}\right) r\right) \\
& =\left(\Pi_{\varphi, \psi}\left(r \pi_{\psi}\left(u_{n}\right) r\right)\right)^{\omega} \\
& =\left(p \pi_{\varphi}\left(u_{n}\right) p\right)^{\omega} \in \mathcal{U}(\mathcal{P}) .
\end{aligned}
$$


Since $\mathcal{F} \subset p \pi_{\varphi}(q) \mathcal{M}$, using Lemma 2.4 (with letting the $Q$ there be the trivial algebra), we obtain $\lim _{n \rightarrow \omega}\left\|\mathrm{E}_{\mathrm{L}_{\varphi}(\mathbf{R})}\left(b^{*} p \pi_{\varphi}\left(u_{n}\right) p a\right)\right\|_{2}=0$ for all $a, b \in \mathcal{F}$. This implies that

$$
\sum_{a, b \in \mathcal{F}}\left\|\mathrm{E}_{\left(\mathrm{L}_{\varphi}(\mathbf{R})\right)^{\omega}}\left(b^{*} \pi_{\varphi^{\omega}}\left(\left(u_{n}\right)^{\omega}\right) p a\right)\right\|_{2}^{2}=\lim _{n \rightarrow \omega} \sum_{a, b \in \mathcal{F}}\left\|\mathrm{E}_{\mathrm{L}_{\varphi}(\mathbf{R})}\left(b^{*} p \pi_{\varphi}\left(u_{n}\right) p a\right)\right\|_{2}^{2}=0 .
$$

Equation (4.5) with $\mathcal{F} \subset p \pi_{\varphi}(q) \mathcal{M}$ and Equation (4.8) give a contradiction. This finishes the proof of the Claim.

Next, for each $i \in\{1,2\}$, put $\mathcal{M}_{i}=\mathrm{c}_{\varphi}\left(M_{i}\right)$. We have $\mathcal{M}=\mathcal{M}_{1} *_{\mathrm{L}_{\varphi}(\mathbf{R})} \mathcal{M}_{2}$ (see [Ue98a, Theorem 5.1]). Observe that since $M_{1}$ is globally invariant under $\sigma^{\psi}$, we have $\Pi_{\varphi, \psi}\left(\mathrm{c}_{\psi}\left(M_{1}\right)\right)=$ $\mathrm{c}_{\varphi}\left(M_{1}\right)=\mathcal{M}_{1}$. Since $r \in \mathrm{L}_{\psi}(\mathbf{R}) \subset \mathrm{c}_{\psi}\left(M_{1}\right)$, we have $p=\Pi_{\varphi, \psi}(r) \in \mathcal{M}_{1}$. Since $Q \cap q M_{1} q$ is diffuse and globally invariant under $\sigma^{\psi_{q}}$, we have $\Pi_{\varphi, \psi}\left(r \mathrm{c}_{\psi_{q}}\left(Q \cap q M_{1} q\right) r\right) \npreceq_{\mathcal{M}} \mathrm{L}_{\varphi}(\mathbf{R})$ by Lemma 2.5. Then [BHR12, Theorem 2.5] implies that $\left(\Pi_{\varphi, \psi}\left(r \mathrm{c}_{\psi_{q}}\left(Q \cap q M_{1} q\right) r\right)\right)^{\prime} \cap p \pi_{\varphi}(q) \mathcal{M} \pi_{\varphi}(q) p \subset$ $p \pi_{\varphi}(q) \mathcal{M}_{1} \pi_{\varphi}(q) p$ and hence

$$
\mathcal{Q}^{\prime} \cap p \pi_{\varphi}(q) \mathcal{M} \pi_{\varphi}(q) p=\mathcal{Q}^{\prime} \cap p \pi_{\varphi}(q) \mathcal{M}_{1} \pi_{\varphi}(q) p .
$$

The set of projections $s \in \mathcal{Q}^{\prime} \cap p \pi_{\varphi}(q) \mathcal{M} \pi_{\varphi}(q) p=\mathcal{Q}^{\prime} \cap p \pi_{\varphi}(q) \mathcal{M}_{1} \pi_{\varphi}(q) p$ such that $\mathcal{Q} s \subset \mathcal{M}_{1} s$ attains its maximum in a unique projection $z$ that belongs to $\mathcal{Z}\left(\mathcal{Q}^{\prime} \cap p \pi_{\varphi}(q) \mathcal{M} \pi_{\varphi}(q) p\right)=$ $\mathcal{Z}\left(\mathcal{Q}^{\prime} \cap p \pi_{\varphi}(q) \mathcal{M}_{1} \pi_{\varphi}(q) p\right)$. Assume by contradiction that $z \neq p \pi_{\varphi}(q)$. Put $z^{\perp}:=p \pi_{\varphi}(q)-z \in$ $\mathcal{Z}\left(\mathcal{Q}^{\prime} \cap p \pi_{\varphi}(q) \mathcal{M} \pi_{\varphi}(q) p\right)$. By assumption, we have $z^{\perp} \neq 0$.

Observe that since $Q \subset q M q$ is a subfactor that is not amenable, $\mathcal{Q}=\Pi_{\varphi, \psi}\left(r \mathrm{c}_{\psi_{q}}(Q) r\right)$ has no amenable direct summand by [BHR12, Proposition 2.8]. By the previous Claim, we moreover have $\mathcal{P} \npreceq \mathcal{M}^{\omega}\left(\mathrm{L}_{\varphi}(\mathbf{R})\right)^{\omega}$. Then Corollary 4.2 implies that there exists $i \in\{1,2\}$ such that $\mathcal{Q} z^{\perp} \preceq \mathcal{M} \mathcal{M}_{i}$. Hence, there exist $n \geq 1$, a finite trace projection $f \in \mathbf{M}_{n}\left(\mathcal{M}_{i}\right)$ (with respect to the canonical trace $\left.\operatorname{Tr}_{\varphi_{i}} \otimes \operatorname{tr}_{n}\right)$, a nonzero partial isometry $v \in\left(z^{\perp} \mathcal{M} \otimes \mathbf{M}_{1, n}(\mathbf{C})\right) f$ and a unital normal $*$-homomorphism $\pi: \mathcal{Q} z^{\perp} \rightarrow f \mathbf{M}_{n}\left(\mathcal{M}_{i}\right) f$ such that $x v=v \pi(x)$ for all $x \in \mathcal{Q} z^{\perp}$. In particular, we have $\Pi_{\varphi, \psi}\left(r c_{\psi_{q}}\left(Q \cap q M_{1} q\right) r\right) v \subset v f \mathbf{M}_{n}\left(\mathcal{M}_{i}\right) f$. Since $\Pi_{\varphi, \psi}\left(r \mathrm{c}_{\psi_{q}}\left(Q \cap q M_{1} q\right) r\right) \npreceq \mathcal{M}$ $\mathrm{L}_{\varphi}(\mathbf{R})$, BHR12, Theorem 2.5] and its Claim imply that $i=1$ and $v \in\left(z^{\perp} \mathcal{M}_{1} \otimes \mathbf{M}_{1, n}(\mathbf{C})\right) f$. Therefore we have $v v^{*} \in \mathcal{Q}^{\prime} \cap p \pi_{\varphi}(q) \mathcal{M}_{1} \pi_{\varphi}(q) p, v v^{*} \neq 0, v v^{*} \leq z^{\perp}$ and $\mathcal{Q}\left(z+v v^{*}\right) \subset(z+$ $\left.v v^{*}\right) \mathcal{M}_{1}\left(z+v v^{*}\right)$. This contradicts the maximality of the projection $z \in \mathcal{Q}^{\prime} \cap p \pi_{\varphi}(q) \mathcal{M}_{1} \pi_{\varphi}(q) p$.

Thus, we have $z=p \pi_{\varphi}(q)$ and hence

$$
\Pi_{\varphi, \psi}\left(r \mathrm{c}_{\psi_{q}}(Q) r\right)=\mathcal{Q} \subset p \pi_{\varphi}(q) \mathcal{M}_{1} \pi_{\varphi}(q) p=\Pi_{\varphi, \psi}\left(r \mathrm{c}_{\psi_{q}}\left(q M_{1} q\right) r\right) .
$$

This implies that $r \mathrm{c}_{\psi_{q}}(Q) r \subset r \mathrm{c}_{\psi_{q}}\left(q M_{1} q\right) r$. Since this holds for every nonzero finite trace projection $r \in \mathrm{L}_{\psi}(\mathbf{R})$, we obtain $\mathrm{c}_{\psi_{q}}(Q) \subset \mathrm{c}_{\psi_{q}}\left(q M_{1} q\right)$. Observe that $\pi_{\psi_{q}}(q M q) \subset \mathrm{c}_{\psi_{q}}(q M q)$ is the fixed point algebra by an action of $\mathbf{R}$, called the dual action of $\sigma^{\psi_{q}}$, (see [Ta03, Theorem X.2.3 (i)]) and hence there exists a (non-normal) conditional expectation $\mathrm{F}: \mathrm{c}_{\psi_{q}}(q M q) \rightarrow$ $\pi_{\psi_{q}}(q M q)$ such that $\mathrm{F}\left(\mathrm{c}_{\psi_{q}}\left(q M_{1} q\right)\right)=\pi_{\psi_{q}}\left(q M_{1} q\right)$. By applying the conditional expectation $\mathrm{F}$ to $\pi_{\psi_{q}}(Q) \subset \mathrm{c}_{\psi_{q}}(Q) \subset \mathrm{c}_{\psi_{q}}\left(q M_{1} q\right)$, we obtain $\pi_{\psi_{q}}(Q) \subset \pi_{\psi_{q}}\left(q M_{1} q\right)$ and hence $Q \subset q M_{1} q$. This finishes the proof of Lemma 4.5.

Proof of Theorem 4.3. Since both $Q$ and $Q \cap M_{1}$ are with expectation in $M$, we may choose a faithful state $\psi \in M_{*}$ such that both $Q$ and $Q \cap M_{1}$ are globally invariant under the modular automorphism group $\sigma^{\psi}$. Denote by $\mathbf{R} \rightarrow \mathcal{U}(M): t \mapsto u_{t}=[D \psi: D \varphi]_{t}$ the Connes RadonNikodym cocycle (see [Co72, Théorème 1.2.1]) satisfying $\sigma_{t}^{\psi}=\operatorname{Ad}\left(u_{t}\right) \circ \sigma_{t}^{\varphi}$ for all $t \in \mathbf{R}$.

Fix any $t \in \mathbf{R}$. Define the unital normal $*$-isomorphism $\pi_{t}: Q \cap M_{1} \rightarrow M: x \mapsto u_{t}{ }^{*} x u_{t}$. Observe that

$$
\pi_{t}\left(Q \cap M_{1}\right)=u_{t}^{*} Q \cap M_{1} u_{t}=u_{t}^{*} \sigma_{t}^{\psi}\left(Q \cap M_{1}\right) u_{t}=\sigma_{t}^{\varphi}\left(Q \cap M_{1}\right) \subset \sigma_{t}^{\varphi}\left(M_{1}\right)=M_{1}
$$


and $x u_{t}=u_{t} \pi_{t}(x)$ for all $x \in Q \cap M_{1}$. Since $Q \cap M_{1} \subset M_{1}$ is diffuse and with expectation, Proposition 2.7 (1) implies that $u_{t} \in \mathcal{U}\left(M_{1}\right)$. Since this holds for every $t \in \mathbf{R}$, we obtain

$$
\sigma_{t}^{\psi}\left(M_{1}\right)=u_{t} \sigma_{t}^{\varphi}\left(M_{1}\right) u_{t}^{*}=u_{t} M_{1} u_{t}^{*}=M_{1} \text {. }
$$

This implies that $\psi=\psi \circ \mathrm{E}_{M_{1}}$ where $\mathrm{E}_{M_{1}}: M \rightarrow M_{1}$ is the unique faithful normal conditional expectation.

Since $Q \cap M_{1} \subset M_{1}$ is diffuse and with expectation, we have $Q^{\prime} \cap M \subset\left(Q \cap M_{1}\right)^{\prime} \cap M=$ $\left(Q \cap M_{1}\right)^{\prime} \cap M_{1}$ by Proposition 2.7 (1) and hence $Q^{\prime} \cap M=Q^{\prime} \cap M_{1}$. Denote by $z \in \mathcal{Z}\left(Q^{\prime} \cap M^{\omega}\right)$ the unique central projection such that $\left(Q^{\prime} \cap M^{\omega}\right) z$ is diffuse and $\left(Q^{\prime} \cap M^{\omega}\right) z^{\perp}$ is atomic. By HR14, Theorem 2.3], we have $z \in \mathcal{Z}\left(Q^{\prime} \cap M\right)=\mathcal{Z}\left(Q^{\prime} \cap M_{1}\right)$ and $\left(Q^{\prime} \cap M^{\omega}\right) z^{\perp}=\left(Q^{\prime} \cap M\right) z^{\perp}=$ $\left(Q^{\prime} \cap M_{1}\right) z^{\perp}$. Observe that $z \in\left(M_{1}\right)^{\psi}$.

Denote by $\left(z_{n}\right)_{n}$ a sequence of central projections in $\mathcal{Z}(Q z)$ such that $\sum_{n} z_{n}=z, Q z_{0}$ has a diffuse center and $Q z_{n}$ is a diffuse factor for all $n \geq 1$. We have $\mathcal{Z}(Q z) \subset(Q z)^{\prime} \cap z M^{\psi} z=$ $z\left(Q^{\prime} \cap M^{\psi}\right) z=z\left(Q^{\prime} \cap\left(M_{1}\right)^{\psi}\right) z$. Moreover, since $\mathcal{Z}(Q z) z_{0} \subset z_{0} M_{1} z_{0}$ is diffuse and globally invariant under the modular automorphism group $\sigma^{\psi_{z_{0}}}$, we have $Q z_{0} \subset\left(\mathcal{Z}(Q z) z_{0}\right)^{\prime} \cap z_{0} M z_{0}=$ $\left(\mathcal{Z}(Q z) z_{0}\right)^{\prime} \cap z_{0} M_{1} z_{0}$ by Proposition 2.7 (1). Finally, for all $n \geq 1$, since $Q z_{n} \subset z_{n} M z_{n}$ is a non type I subfactor that is globally invariant under the modular automorphism group $\sigma^{\psi_{z_{n}}}$ and such that $Q z_{n} \cap z_{n} M_{1} z_{n}=\left(Q \cap M_{1}\right) z_{n}$ and $\left(Q z_{n}\right)^{\prime} \cap\left(z_{n} M z_{n}\right)^{\omega}=\left(Q^{\prime} \cap M^{\omega}\right) z_{n}$ are diffuse, Lemma 4.4, in the case when $Q z_{n}$ is amenable, and Lemma 4.5, in the case when $Q z_{n}$ is nonamenable, imply that $Q z_{n} \subset z_{n} M_{1} z_{n}$. Therefore, we have $Q z \subset z M_{1} z$. This finishes the proof of Theorem 4.3.

We can finally deduce the main results of this paper.

Proof of Theorem $A$. By applying Theorem 4.3 to the case when the projection $z \in \mathcal{Z}\left(Q^{\prime} \cap M^{\omega}\right)$ satisfies $z=1$, we obtain $Q \subset M_{1}$.

Proof of Corollary $\mathbb{B}$. Since both $Q$ and $Q \cap M_{1}$ are with expectation and $Q \cap M_{1}$ is diffuse, using Lemma 2.1, we may choose a faithful state $\psi \in M_{*}$ such that both $Q$ and $Q \cap M_{1}$ are globally invariant under the modular automorphism group $\sigma^{\psi}$ and the centralizer $\left(Q \cap M_{1}\right)^{\psi}$ is diffuse. Note that by the proof of Theorem $4.3, M_{1}$ is also globally invariant under the modular automorphism group $\sigma^{\psi}$. Next, choose a diffuse abelian von Neumann subalgebra with separable predual $A \subset\left(Q \cap M_{1}\right)^{\psi}$.

Let $x \in Q$ be any element. Denote by $Q_{0} \subset M$ the von Neumann subalgebra generated by the set $\left\{\sigma_{t}^{\psi}(y): t \in \mathbf{R}, y=x\right.$ or $\left.y \in A\right\}$. Observe that $Q_{0} \subset M$ has separable predual and is globally invariant under the modular automorphism group $\sigma^{\psi}$. Since $Q$ is amenable and $Q_{0} \subset Q$ is with expectation, it follows that $Q_{0}$ is also amenable. (It is true even in the nonseparable case that amenability implies injectivity. See Co76].) Since $A \subset\left(Q_{0} \cap M_{1}\right)^{\psi}$ and since $A$ is diffuse, $\left(Q_{0} \cap M_{1}\right)^{\psi}$ is diffuse and so is $Q_{0} \cap M_{1}$ (see e.g. [Bl06, Theorem IV.2.2.3]).

Since $Q_{0}$ is diffuse, amenable and with separable predual, the central sequence algebra $Q_{0}^{\prime} \cap Q_{0}^{\omega}$ is diffuse (see e.g. [Ho14, Proposition 2.6]). Since $Q_{0} \subset M$ is with expectation, the inclusion $Q_{0}^{\prime} \cap Q_{0}^{\omega} \subset Q_{0}^{\prime} \cap M^{\omega}$ is with expectation and hence $Q_{0}^{\prime} \cap M^{\omega}$ is diffuse. Since $Q_{0} \cap M_{1}$ is moreover diffuse and with expectation, we obtain that $Q_{0} \subset M_{1}$ by Theorem $\AA$ and hence $x \in M_{1}$. Since this holds true for all $x \in Q$, we deduce $Q \subset M_{1}$.

\section{Appendix A. Bicentralizer problem for free product von Neumann algebras}

Let $(M, \varphi)$ be any $\sigma$-finite von Neumann algebra endowed with a faithful normal state. Following [Ha85, the asymptotic centralizer of $\varphi$ is defined by

$$
\operatorname{AC}(M, \varphi)=\left\{\left(x_{n}\right)_{n} \in \ell^{\infty}(\mathbf{N}, M): \lim _{n \rightarrow \infty}\left\|x_{n} \varphi-\varphi x_{n}\right\|=0\right\}
$$


and the bicentralizer of $\varphi$ is defined by

$$
\mathrm{B}(M, \varphi)=\left\{a \in M: \lim _{n \rightarrow \infty}\left\|a x_{n}-x_{n} a\right\|_{\varphi}=0, \forall\left(x_{n}\right)_{n} \in \mathrm{AC}(M, \varphi)\right\} .
$$

Haagerup showed in $\mathrm{Ha} 85$, that any amenable type $\mathrm{III}_{1}$ factor with separable predual has trivial bicentralizer. It is an open problem, known as Connes's bicentralizer problem, to decide whether any type $\mathrm{III}_{1}$ factor with separable predual has trivial bicentralizer.

It was recently showed in [HI15, Proposition 3.3] that $\mathrm{B}(M, \varphi)=\left(\left(M^{\omega}\right)^{\varphi^{\omega}}\right)^{\prime} \cap M$ for every nonprincipal ultrafilter $\omega \in \beta(\mathbf{N}) \backslash \mathbf{N}$. Using this characterization, we give a short proof of an unpublished result due to the second named author showing that Connes's bicentralizer problem has a positive solution for all type $\mathrm{III}_{1}$ free product factors.

For each $i \in\{1,2\}$, let $\left(M_{i}, \varphi_{i}\right)$ be any nontrivial $\sigma$-finite von Neumann algebra endowed with a faithful normal state. Assume moreover that $\operatorname{ker}\left(\sigma^{\varphi_{1}}\right) \cap \operatorname{ker}\left(\sigma^{\varphi_{2}}\right)=\{0\}$. Denote by $(M, \varphi)=\left(M_{1}, \varphi_{1}\right) *\left(M_{2}, \varphi_{2}\right)$ the free product. By [Ue10, Theorem 4.1], we have $M=M_{c} \oplus M_{d}$ where $M_{c}$ is a type $\mathrm{III}_{1}$ factor and $M_{d}=0$ or $M_{d}$ is a multimatrix algebra. Put $\varphi_{c}=\left.\frac{1}{\varphi\left(1_{M_{c}}\right)} \varphi\right|_{M_{c}}$.

Theorem A.1. Keep the same notation as above. Then $\mathrm{B}\left(M_{c}, \varphi_{c}\right)=\mathbf{C} 1_{M_{c}}$.

Proof. In the case when both $M_{1}$ and $M_{2}$ are atomic, $\varphi_{c}$ is an almost periodic state such that $\left(\left(M_{c}\right)^{\varphi_{c}}\right)^{\prime} \cap M_{c}^{\omega}=\mathbf{C} 1_{M_{c}}$ by [Ue11, Theorem 2.2]. Then we have $\mathrm{B}\left(M_{c}, \varphi_{c}\right) \subset\left(\left(M_{c}\right)^{\varphi_{c}}\right)^{\prime} \cap M_{c}=$ $\mathrm{C} 1_{M_{c}}$.

Next, we may assume that $M_{1}$ has a diffuse direct summand. Since $M_{c}$ is of type III and using Ue10, Lemma 2.2], up to cutting down $M$ by the central projection in $M_{1}$ that supports the diffuse direct summand of $M_{1}$, we may assume without loss of generality that $M_{1}$ is diffuse. In that case, we have $M=M_{c}$. Observe that $M_{1}^{\omega}$ and $M_{2}^{\omega}$ are both globally invariant under the modular automorphism group $\sigma^{\varphi^{\omega}}$ and are $*$-free inside $M^{\omega}$ with respect to the state $\varphi^{\omega}$ (see [Ue00, Proposition 4]). Letting $P=M_{1}^{\omega} \vee M_{2}^{\omega}$, we have $\left(P,\left.\varphi^{\omega}\right|_{P}\right) \cong\left(M_{1}^{\omega}, \varphi_{1}^{\omega}\right) *\left(M_{2}^{\omega}, \varphi_{2}^{\omega}\right)$ and $M \subset P \subset M^{\omega}$.

Since $M_{1}$ is diffuse and $M_{2} \neq \mathbf{C} 1$, we have that $\left(M_{1}^{\omega}\right)^{\varphi_{1}^{\omega}}$ is diffuse and $\left(M_{2}^{\omega}\right)^{\varphi_{2}^{\omega}} \neq \mathbf{C} 1$ by Proposition 2.8. Using [HI15, Proposition 3.3] and Proposition 2.7 (1), we have

$$
\mathrm{B}(M, \varphi)=\left(\left(M^{\omega}\right)^{\varphi^{\omega}}\right)^{\prime} \cap M \subset\left(\left(M_{1}^{\omega}\right)^{\varphi_{1}^{\omega}}\right)^{\prime} \cap P \cap M \subset\left(\left(M_{1}^{\omega}\right)^{\varphi_{1}^{\omega}}\right)^{\prime} \cap M_{1}^{\omega} \cap M \subset M_{1} .
$$

Next, one can choose an invertible element $w \in\left(M_{2}^{\omega}\right)_{2}^{\omega}$ such that $\varphi_{2}^{\omega}(w)=0$. For all $y \in$ $\mathrm{B}(M, \varphi) \subset M_{1}$ such that $\varphi_{1}(y)=0$, using the freeness with respect to $\varphi^{\omega}$ and since $y w=w y$, we have

$$
\varphi^{\omega}\left(w^{*} y^{*} y w\right)=\varphi^{\omega}\left(w^{*} y^{*} w y\right)=0 .
$$

Therefore $w^{*} y^{*} y w=0$ and hence $y=0$ since $w$ is invertible. It immediately follows that $\mathrm{B}(M, \varphi)=\mathrm{C} 1$. This finishes the proof of Theorem A.1.

\section{REFERENCES}

[AH12] H. Ando, U. HaAgerup, Ultraproducts of von Neumann algebras. J. Funct. Anal. 266 (2014), 68426913.

[BC14] R. Boutonnet, A. CARderi, Maximal amenable von Neumann subalgebras arising from maximal amenable subgroups. Geom. Funct. Anal. 25 (2015), 1688-1705.

[BHR12] R. Boutonnet, C. Houdayer, S. Raum, Amalgamated free product type III factors with at most one Cartan subalgebra. Compos. Math. 150 (2014), 143-174.

[B106] B. Blackadar, Operator Algebras. Encyclopaedia of Mathematical Sciences, 122. Operator Algebras and Non-commutative Geometry, 3. Springer-Verlag, Berlin, 2006. xx+517 pp.

[CH08] I. Chifan, C. HoudayeR, Bass-Serre rigidity results in von Neumann algebras. Duke Math. J. 153 (2010), 23-54.

[Co72] A. Connes, Une classification des facteurs de type III. Ann. Sci. École Norm. Sup. 6 (1973), 133-252.

[Co75] A. Connes, Classification of injective factors. Cases $\mathrm{II}_{1}, \mathrm{II}_{\infty}, \mathrm{III}_{\lambda}, \lambda \neq 1$. Ann. of Math. 74 (1976), $73-115$. 
[Co76] A. Connes, On the cohomology of operator algebras. J. Funct. Anal. 28 (1978), 248-253.

[CS78] A. Connes, E. Størmer, Homogeneity of the state space of factors of type $\mathrm{III}_{1}$. J. Funct. Anal. 28 (1978), 187-196.

[FM75] J. Feldman, C.C. Moore, Ergodic equivalence relations, cohomology, and von Neumann algebras. I and II. Trans. Amer. Math. Soc. 234 (1977), 289-324, 325-359.

[Ha85] U. HAAGERUP, Connes' bicentralizer problem and uniqueness of the injective factor of type $\mathrm{III}_{1}$. Acta Math. 69 (1986), 95-148.

[HS90] U. HAAGERUP, E. STøRMER, Equivalence of normal states on von Neumann algebras and the flow of weights. Adv. Math. 83 (1990), 180-262.

[Ho12a] C. Houdayer, A class of $\mathrm{II}_{1}$ factors with an exotic abelian maximal amenable subalgebra. Trans. Amer. Math. Soc. 366 (2014), 3693-3707.

[Ho12b] C. HoudayeR, Structure of $\mathrm{II}_{1}$ factors arising from free Bogoljubov actions of arbitrary groups. Adv. Math. 260 (2014), 414-457.

[Ho14] C. Houdayer, Gamma stability in free product von Neumann algebras. Commun. Math. Phys. 336 (2015), 831-851.

[HI15] C. HoudAYeR, Y. IsOno, Unique prime factorization and bicentralizer problem for a class of type III factors. arXiv: 1503.01388

[HR14] C. Houdayer, S. Raum, Asymptotic structure of free Araki-Woods factors. Math. Ann. 363 (2015), 237-267.

[HR10] C. HoudAyer, É. RicARD, Approximation properties and absence of Cartan subalgebra for free ArakiWoods factors. Adv. Math. 228 (2011), 764-802.

[HU15] C. Houdayer, Y. Ueda, Rigidity of free product von Neumann algebra. To appear in Compos. Math. arXiv: 1507.02157

[HV12] C. Houdayer, S. Vaes, Type III factors with unique Cartan decomposition. J. Math. Pures Appl. 100 (2013), 564-590.

[Io12] A. IoAnA, Cartan subalgebras of amalgamated free product $\mathrm{II}_{1}$ factors. Ann. Sci. École Norm. Sup. 48 (2015), 71-130.

[IPP05] A. Ionna, J. Peterson, S. Popa, Amalgamated free products of w-rigid factors and calculation of their symmetry groups. Acta Math. 200 (2008), 85-153.

[Jo82] V.F.R. Jones, Index for subfactors. Invent. Math. 72 (1983), 1-25.

[Ka82] R.V. Kadison, Diagonalizing matrices. Amer. J. Math. 106 (1984), 1451-1468.

[Ko88] H. Kosaki, Characterization of crossed product (properly infinite case). Pacific J. Math. 137 (1989), 159-167.

[Kr75] W. Krieger, On ergodic flows and the isomorphism of factors. Math. Ann. 223 (1976), 19-70.

[MU12] M. Martín, Y. UEdA, On the geometry of von Neumann algebra preduals. Positivity 18 (2014), 519-530.

[MT13] T. Masuda, R. Tomatsu, Classification of actions of discrete Kac algebras on injective factors. To appear in Mem. Amer. Math. Soc. arXiv:1306.5046

[Oc85] A. OCNEANu, Actions of discrete amenable groups on von Neumann algebras. Lecture Notes in Mathematics, 1138. Springer-Verlag, Berlin, 1985. iv+115 pp.

[Oz15] N. Ozawa, A remark on amenable von Neumann subalgebras in a tracial free product. Proc. Japan Acad. Ser. A Math. Sci. 91 (2015), 104.

[Pe06] J. Peterson, $\mathrm{L}^{2}$-rigidity in von Neumann algebras. Invent. Math. 175 (2009), 417-433.

[PP84] M. Pimsner, S. PopA, Entropy and index for subfactors. Ann. Sci. École Norm. Sup. 19 (1986), $57-106$.

[Po83] S. PopA, Maximal injective subalgebras in factors associated with free groups. Adv. Math. 50 (1983), $27-48$.

[Po90] S. PopA, Markov traces on universal Jones algebras and subfactors of finite index. Invent. Math. 111 (1993), 375-405.

[Po01] S. POPA, On a class of type $\mathrm{II}_{1}$ factors with Betti numbers invariants. Ann. of Math. 163 (2006), 809-899.

[Po03] S. POPA, Strong rigidity of $\mathrm{II}_{1}$ factors arising from malleable actions of w-rigid groups I. Invent. Math. 165 (2006), 369-408.

[Po06] S. POPA, On the superrigidity of malleable actions with spectral gap. J. Amer. Math. Soc. 21 (2008), 981-1000.

[Ta02] M. TAKeSAKI, Theory of Operator Algebras. I. Encyclopedia of Mathematical Sciences, 124, Operator Algebras and Non-commutative Geometry, 5, Springer, Berlin, 2002. xx+415 pp.

[Ta03] M. TAKESAKI, Theory of operator algebras. II. Encyclopaedia of Mathematical Sciences, 125. Operator Algebras and Non-commutative Geometry, 6. Springer-Verlag, Berlin, 2003. xxii+518 pp.

[Ue98a] Y. UEDA, Amalgamated free products over Cartan subalgebra. Pacific J. Math. 191 (1999), 359-392. 
[Ue98b] Y. UEDA, Remarks on free products with respect to non-tracial states. Math. Scand. 88 (2001), 111-125.

[Ue00] Y. UEDA, Fullness, Connes' $\chi$-groups, and ultra-products of amalgamated free products over Cartan subalgebras. Trans. Amer. Math. Soc. 355 (2003), 349-371.

[Ue10] Y. UEDA, Factoriality, type classification and fullness for free product von Neumann algebras. Adv. Math. 228 (2011), 2647-2671.

[Ue11] Y. UEDA, On type $\mathrm{III}_{1}$ factors arising as free products. Math. Res. Lett. 18 (2011), 909-920.

[Ue12] Y. UEDA, Some analysis on amalgamated free products of von Neumann algebras in non-tracial setting. J. London Math. Soc. 88 (2013), 25-48.

[Va06] S. VAES, Rigidity results for Bernoulli actions and their von Neumann algebras (after Sorin Popa). Astérisque 311 (2007), 237-294.

[Va07] S. VAEs, Explicit computations of all finite index bimodules for a family of $\mathrm{II}_{1}$ factors. Ann. Sci. École Norm. Sup. 41 (2008), 743-788.

[Vo85] D.-V. Voiculescu, Symmetries of some reduced free product $C^{*}$-algebras. Operator algebras and Their Connections with Topology and Ergodic Theory, Lecture Notes in Mathematics 1132. Springer-Verlag, (1985), 556-588.

[VDn92] D.-V. Voiculescu, K.J. Dykema, A. NicA, Free random variables. CRM Monograph Series 1. American Mathematical Society, Providence, RI, 1992.

Laboratoire de Mathématiques d’Orsay, Université Paris-Sud, CNRS, Université Paris-Saclay, 91405 ORSAY, FRANCE

E-mail address: cyril.houdayer@math.u-psud.fr

Graduate School of Mathematics, Kyushu University, Fukuoka, 810-8560, JAPAN

E-mail address: ueda@math.kyushu-u.ac.jp 